\title{
Upscaling of fractured oil reservoirs using homogenization including non-equilibrium capillary pressure and relative permeability
}

\author{
Hamidreza Salimi • Johannes Bruining
}

Received: 19 January 2011 / Accepted: 8 November 2011 / Published online: 23 December 2011

(C) The Author(s) 2011. This article is published with open access at Springerlink.com

\begin{abstract}
Recovery from incompletely water-wet fractured reservoirs can be extremely low. A reason for the low recovery is related to wetting issues, whereas the reason for slow recovery can be the non-equilibrium behavior of capillary pressure. One of the non-equilibrium theories is developed by Barenblatt et al. and it modifies both capillary pressure and relative permeabilities. The other theory is developed by Hassanizadeh et al. and it only deals with non-equilibrium effects for capillary pressure. To incorporate non-equilibrium in larger-scale problems, we apply homogenization to derive an upscaled model for fractured reservoirs in which the nonequilibrium effects are included. We formulate a fully implicit three-dimensional upscaled numerical model. Furthermore, we develop a computationally efficient numerical approach to solve the upscaled model. We use simulations to determine the range of delay times and capillary-damping coefficients for which discernable effects occur in terms of oil recovery. It is shown that at low Peclet numbers, i.e., when the residence time of the fluids in the fracture is long with respect to the imbibition time, incorporation of delay times of the order of few months have no significant effect on the oil recovery. However, when the Peclet number is large, the delay times reduce the rate of oil recovery. We discuss for which values of the delay time (Barenblatt) and capillary-damping coefficient (Hassanizadeh), significant delays in oil production occur.
\end{abstract}

H. Salimi $(\varangle) \cdot$ J. Bruining

Department of Geotechnology, Delft University

of Technology, Delft, The Netherlands

e-mail: h.salimi@tudelft.nl

J. Bruining

e-mail: j.bruining@tudelft.nl
Keywords Upscaling • Non-equilibrium effects • Fractured reservoirs • Oil recovery $\cdot$ Homogenization

\section{Introduction}

Fractured hydrocarbon reservoirs provide over $20 \%$ of the world's oil reserves and production [16, 41]. Virtually, all reservoirs contain at least some natural fractures [1, 32]. However, from the point of view of reservoir modeling, a fractured reservoir is defined as a reservoir in which naturally occurring fractures have a significant effect on fluid flow [42-46]. We only consider reservoirs where fluid flow occurs predominantly in a connected-fracture network and do not consider the case of limited connectivity and cases in which fractures act as a barrier to fluid flow. Fractured-reservoir simulations completely differ from conventional-reservoir simulations. The challenge of upscaling is to give an accurate representation of the interaction between fractures and matrix blocks.

Many geological situations lead to some type of fractured reservoirs [1, 32]. From the geological point of view, fractured reservoirs can exhibit a number of topologically different configurations. These are reservoirs built from (1) matrix blocks that are bounded by fracture planes in all directions (totally fractured reservoirs, TFRs, or sugar cubes), (2) matrix blocks that are bounded only by more or less vertical fracture planes (vertically fractured reservoirs, VFRs), and (3) matrix blocks that form a connected domain interdispersed with fractures (partially fractured reservoirs, PFRs). Horizontal fractures in the sugar-cube model have a zero or relatively narrow aperture to reflect maximum closure of fractures normal to the maximum component 
of burial stress, which is usually vertical [32], except in shallow hydrology applications [12, 64]. The sugar cube model can still be relevant when the two fracture sets are oblique leading to horizontal columns. If these columns are intersected by a third vertical fracture set, matrix blocks can occur that are bounded by three fracture planes [15, 57]. In the VFR configuration, the top and bottom of the columns are bounded by the impermeable cap- and base-rock.

The current literature in the petroleum community dealing with flow in fractured reservoirs is largely confined to topological equivalents of the sugar-cube model, i.e., the matrix blocks are surrounded by fractures from all sides $[2,3,8,26,27,48,53,61]$. However, the VFR (aggregated-column) model is topologically different because it is not connected to the fracture network through the top and bottom of the matrix column. To our knowledge, the current reservoir simulators have no option to deal with this situation and this is another innovative aspect of this contribution. The novel feature of the upscaled-VFR model is that, for example, fluid may enter the matrix column at one height, travel downward for some time, and then reenter the fractures at a lower height.

In fractured reservoirs, capillary forces, leading to counter-current or co-current imbibition [11, 22, 25, $36,40]$, are the main drivers for waterdrive recovery from the matrix blocks [16]. Reservoir wettability and its effect on oil recovery have been the subject of numerous studies [4, 18, 30, 49, 56]. However, these studies address drainage and imbibition behavior on a laboratory scale, which describe only one facet of multiphase flow in fractured reservoirs. Experimental evidence $[14,34,35,51,52,54,56,58,59,63]$ supports a more general description of capillary pressure and relative permeability functions, which includes a nonequilibrium effect. Reservoir interpretation that does not recognize the potential for reduced recovery because of non-equilibrium effects may lead to an overestimation of the recovery. The concept of the nonequilibrium effect was first introduced by Barenblatt and his colleagues $[6,7,9,10]$. It is equivalent to an alternative formulation introduced by Hassanizadeh and Gray [19, 20] and Pavone [33].

In our previous paper [45], we have derived an upscaled model for vertically fractured reservoirs without including the non-equilibrium effects. In this work, we extend our previous work [45] on upscaling waterdrive recovery in fractured reservoirs to include nonequilibrium effects in capillary pressures and relative permeabilities. We follow both the formulation by Barenblatt, which has been worked out for both capillary-pressure and relative-permeability functions, and the approach by Hassanizadeh, which only includes capillary pressure. To examine for which values of the parameters non-equilibrium effects have a significant effect on larger-scale problems, we construct an upscaled model in which the non-equilibrium effects are included for the matrix columns. We use simulations to determine the range of delay times and capillary damping coefficients that have discernable effects on oil recovery.

The objective of this paper is (1) the construction of an upscaled model in which the non-equilibrium effects are included; (2) comparison of Barenblatt's and Hassanizadeh's approach for non-equilibrium capillary pressures in upscaled waterdrive recovery from fractured reservoirs on the field scale; (3) development of an efficient fully implicit numerical method solving the upscaled equations; and (4) quantifying conditions for which non-equilibrium capillary-pressure effects determine the recovery behavior.

The paper is organized as follows: first, we briefly describe the physical model and the topological configuration for vertically fractured reservoirs. Next, we explain the theory of non-equilibrium effects in twophase flow. Thirdly, we elucidate the upscaled model. Subsequently, we derive the fully implicit numerical scheme. After that, we introduce the Peclet number to distinguish two different regimes and consider the effect of gravity. Then, we provide numerical simulations at the field scale to study the mechanisms mentioned above. Finally, we summarize our findings in the conclusions Section.

\section{Physical model}

In this paper, we extend our previous work [44-46] on upscaling waterdrive recovery in fractured reservoirs to include non-equilibrium effects in capillary pressures and relative permeabilities. The two mostused non-equilibrium theories are due to Barenblatt and Hassanizadeh. We follow both the formulation by Barenblatt, which has been worked out for both the capillary-pressure and the relative-permeability functions, and the approach by Hassanizadeh, which only includes the capillary pressure. Here, we only consider the non-equilibrium effects for the matrix-column system and not for the fracture system. This is related to the fact that the non-equilibrium effects for fractures, due to their high permeabilities and consequently low capillary forces, are negligible. Each theory introduces a new equation to the conventional two-phase flow system of equations to describe the non-equilibrium effects, and subsequently a 
new primary variable. In Barenblatt's theory, the new primary variable is the effective saturation and in Hassanizadeh's theory, the new primary variable is the dynamic capillary pressure. For reasons of easy reference, we shortly repeat the theory for obtaining the upscaled equations.

We consider a vertically fractured network (Fig. 1) in the domain $Q=A_{Q} \times Z$, where $A_{Q}=\{x, y \in \mathrm{R} \mid 0 \leq$ $x \leq L, 0 \leq y \leq W\}$ and $Z=\{z \in \mathrm{R} \mid 0 \leq z \leq H\}$, where $\mathrm{R}$ represents the set of real numbers. We use $L, W$, and $H$ to denote the length, width and height of the fractured reservoir. There are two mutually perpendicular vertical fracture sets $F_{1}, F_{2}$, which surround the matrix column $B_{m}=\Omega_{m} \times Z$, where $\Omega_{m}$ (see Fig. 1) denotes a domain of a horizontal cross-section of a matrix column. Therefore, matrix columns are connected from the top to the bottom of the reservoir domain and there is no direct horizontal fracture connection between the matrix columns. Thus, the matrix columns can capture phase segregation caused by gravity. Half of the fracture space surrounding the matrix columns occupy the domain $B_{f}=\Omega_{f} \times Z$. Furthermore, $B=\Omega \times Z$ denotes the small periodic units that occupy the domain $B=B_{f} \cup B_{m}$. All the small units together constitute the VFR.

We simulate injection of water into a vertically fractured oil reservoir. The inclusion of the nonequilibrium effects modifies the boundary at the interface between fracture and matrix. We apply continuity of capillary pressure and continuity of fluid flow as boundary conditions on the vertical interfaces between fractures and matrix columns. Flux continuity follows from fluid conservation at the interface between fracture and matrix. There is capillary pressure continuity at the boundary of the fractures and matrix columns unless one of the phases either in the matrix or in the fracture is immobile [60]. Indeed, when one of the phases is immobile, the pressure of that phase depends on local conditions and cannot be determined globally.
Hence, the capillary pressure, which is the difference between the phase pressure of the non-wetting phase and wetting phase, can be discontinuous. However, as residual saturations do not flow, this presents no difficulties for modeling. Continuity of force, and hence continuity of phase pressures, implies continuity of capillary pressure when both phases are mobile.

Moreover, we incorporate gravity directly inside the matrix columns. As a result, there is a net flow within the matrix columns. The symmetry of the fracture pattern in the horizontal plane is such that the fracture permeability can be considered isotropic. We only consider two-phase (oil and water) incompressible flow where the water viscosity, $\mu_{w}$, and the oil viscosity, $\mu_{o}$, are assumed to be constant.

\subsection{Non-equilibrium effects}

The classical two-phase flow model proposed by Muskat and Meres [31], and Leverett [29] is based upon the fundamental assumption that the local state of the flow is in equilibrium. This means that the two-phase functions $k_{r w}, k_{r o}$, and Leverett $J$-function are only functions of the water saturation $S_{w}$, independent of whether the water saturation decreases or increases. Therefore, at local equilibrium we have

$k_{r w}=k_{r w}\left(S_{w}\right), \quad k_{r o}=k_{r o}\left(S_{w}\right)$,

$P_{c}\left(S_{w}\right)=\sigma_{w o} \sqrt{\frac{\varphi}{k}} J\left(S_{w}\right)$,

where $\sigma_{w o}$ is the oil-water interfacial surface tension, and $\varphi$ and $k$ are, respectively, the porosity and the absolute permeability of the rock. The dimensionless Leverett $J$-function is often assumed to be independent of the porosity and permeability for a specific litho-type.

For increasing (wetting) water saturation, the wetting phase will flow initially in the larger pores, but
Fig. 1 Vertically fractured network (left), matrix column (middle), and horizontal cross section (right) of a small unit that consists of a matrix part and a fracture part

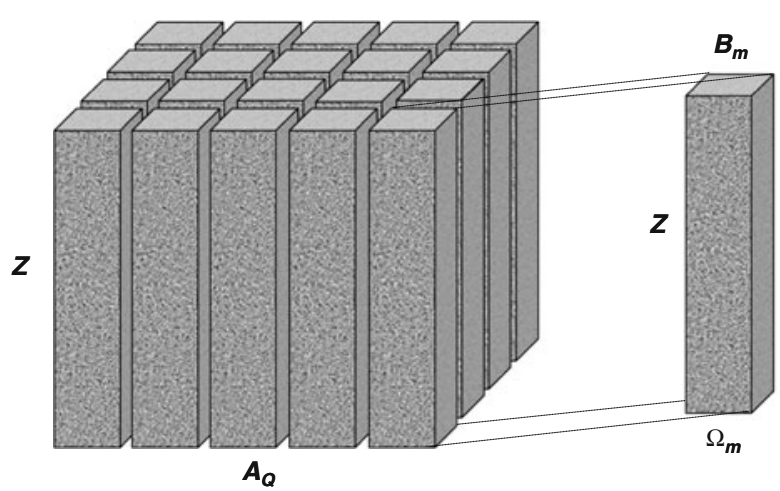


when the steady state $\left(\partial S_{w} / \partial t=0\right)$ is attained, it withdraws in the narrower pores and corners and Eq. 1 becomes applicable. There are two reasons that water initially flows in the larger pores. First, the "permeability" of a cylindrical pore is proportional to the square of the pore radius $R$, whereas the capillary-driving force is inversely proportional to $R$. Hence, even if capillary forces are smaller in larger pores, the velocity is still higher due to the higher permeability. The other reason is that the pores that were originally filled with oil will maintain an oil film at the wall, which is only slowly removed $[23,24]$. In other words, a water-wet medium will effectively be oil-wet in the early stages of imbibition. The slow removal rate of an oil film shows itself in experiments on contact angle measurements. For water-wet media with a finite contact angle, it is possible that the delay time is a few months. This effect is observed in many spontaneous imbibition experiments [14, 34, 35, 51, 52, 54, 56, 58, 59, 63]. During the transition, the wetting-fluid relative permeability is higher than in steady-state conditions. Similarly, during the redistribution of the flow paths both the relative permeability of the non-wetting fluid and the capillary pressure are lower than in steady-state flow. Strictly speaking, this reasoning implies that the relative permeability and capillary pressure functions obtained in steady-state flow experiments cannot be used in transient processes whose characteristic transition times are comparable with characteristic fluid-redistribution times. However, due to the monotonous behavior of these functions, (see Fig. 2), they can still be used to characterize transient flow by evaluating the functions at some effective water saturations. Indeed, the relative permeabilities and the capillary pressure are evaluated not at the actual instantaneous values of the saturation $S_{w}$, but at some effective saturation $\eta \geq S_{w}$, see Fig. 2 .

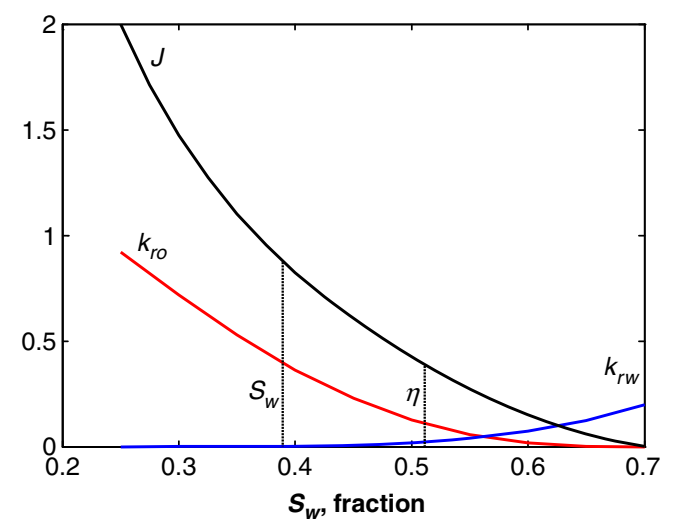

Fig. 2 The effective saturation $\eta$ is always higher than the actual water saturation $S_{w}$
The effective saturation $\eta$ is always larger than the water saturation $S_{w}$ or equal to it when steady state is attained. The concept of effective saturation was first introduced by Barenblatt and his colleagues $[6,7,9,10]$. It is equivalent to an alternative formulation introduced by Hassanizadeh and Gray [20] (see also [21]).

In general, the effective saturation $\eta$ can be different for both the relative permeability curves $\left(k_{r \alpha}\right)$ and the Leveret $J$-function $(J)$. However, following Barenblatt [6], Barenblatt and Gilman [7], and Barenblatt et al. [10], we assume that the effective saturation is the same for all three functions. Thus, instead of the relationships in Eq. 1 we have

$k_{r w}=k_{r w}(\eta), \quad k_{r o}=k_{r o}(\eta), \quad P_{c}(\eta)=\sigma_{w o} \sqrt{\frac{\varphi}{k}} J(\eta)$,

where the functions $k_{r w}, k_{r o}$, and $J$ are the same functions as in Eq. 1.

Due to non-equilibrium effects, the relationship between $\eta$ and $S_{w}$ must be a time-dependent function. We use the hypothesis $[6,9,10]$ that there is a relationship between the local effective water saturation $\eta$ and the actual water saturation $S_{w}$ and its rate of change $\partial S_{w} / \partial t$. Dimensional analysis suggests that such a relationship must include a characteristic redistribution time. Further, linearization of this relationship yields

$\tau_{b} \frac{\partial S_{w}}{\partial t}=\eta-S_{w}$

Here, $\tau_{b}$ is a coefficient having the dimension of (delay) time. If the effective water saturation $\eta$ were fixed and $\tau_{b}$ were constant, then the difference $(\eta-S)$ would decay exponentially as $\exp \left(-t / \tau_{b}\right)$. Therefore, $\tau_{b}$ is a characteristic relaxation time (delay time) needed for the rearrangement of the flow paths to a new steadystate configuration and for the removal of the oil film. Note that in spontaneous imbibition, such a steady state is never achieved. Indeed, the redistribution time, $\tau_{b}$, may depend on the saturation, but we assume that $\tau_{b}$ is constant.

Hassanizadeh and Gray $[19,20]$ derived an alternative theory to deal with non-equilibrium effects based on non-equilibrium thermodynamics. The main constitutive hypothesis in their theory is the dependence of the Helmholtz-free-energy functions for the phases and interfaces on state variables such as mass density, temperature, saturation, porosity, interfacial area, and the solid-phase strain tensor. Explicit inclusion of interfaces and interfacial properties in the macroscale theory of the two-phase flow is an essential characteristic of Hassanizadeh's theory. In this approach, 
the macroscopic capillary pressure is defined solely as an intrinsic property of the system and is not simply equal to the difference in fluid-phase pressures. Nonequilibrium thermodynamics shows that the difference in fluid pressures is not only dependent on saturation, but also on the rate of change of saturation. Their result using a linear approximation is

$P_{c}^{d y n}-P_{c}^{s t a t}=\tau_{h} \frac{\partial S_{o}}{\partial t}$,

where $P_{c}^{d y n}$ denotes the difference between nonwettingphase pressure $\left(P_{n}\right)$ and wetting-phase pressure $\left(P_{w}\right)$, which is the commonly measured quantity. $P_{c}^{\text {stat }}$ denotes the equilibrium (or "static") capillary pressure, and $\tau_{h}$ is a capillary-damping coefficient that may still depend on saturation. Note that $\tau_{h}$ is non-negative, because heat dissipation is always positive. Equation 4 suggests that at a given point in the system and at any given time, the saturation will change locally in order to restore equilibrium; in other words at equilibrium there is equivalence between $\left(P_{n}-P_{w}\right)$ and $P_{c}^{\text {stat }}$. If $\tau_{h}$ is found to be small, equivalence between $P_{c}^{d y n}$ and $P_{c}^{\text {stat }}$ will be re-established instantaneously after equilibrium is disturbed. For porous media with a high permeability (e.g., fractures), the non-equilibrium effect is less pronounced. As permeability decreases, the applicability of the non-equilibrium concept becomes necessary.

The non-equilibrium theory of capillary pressure of Hassanizadeh and Gray versus Barenblatt cannot be related if constant values of $\tau_{b}$ and $\tau_{h}$ were used. Hassanizadeh and Gray [19, 20] do not deal with relative permeabilities, but their theory is based on fundamental non-equilibrium thermodynamic principles, whereas the theory of Barenblatt et al. $[9,10]$ is based on insight that initially larger pores will be filled with water before the water withdraws into the smaller pores. If we would have a linear saturation dependence of the capillary pressure, e.g.,

$P_{c}^{\text {stat }}=P_{c}^{\circ}+\Lambda\left(1-S_{o r}-S_{w}\right)$,

we can relate the delay time $\tau_{b}$ to the capillary-damping coefficient $\tau_{h}$ as

$\Lambda \tau_{b}=\tau_{h}$,

where $\Lambda$ is a constant coefficient. In spite of the fact that the capillary pressure is a nonlinear function of the saturation, we still use this as a basis for comparisons. Note that the linear relationship is only used to connect Barenblatt's theory with Hassanizadeh's theory. For non-linear capillary pressure, we use the average capillary derivative for calculating $\Lambda$.
To the best of our knowledge, no attempt has yet been made to investigate for which values of $\tau_{h}$ or $\tau_{b}$, non-equilibrium effects in fractured reservoirs become significant. In this work, we only consider the nonequilibrium effects for the matrix columns because the important process (i.e., capillary imbibition) occurs in the matrix columns and because the matrix system has a low permeability with respect to the fracture permeability.

The system of Eqs. 2 and 3 for Barenblatt's approach and Eq. 4 for Hassanizadeh's approach can be straightforwardly added to the conventional two-phase flow system of equations to constitute the basic mathematical model for our further consideration of the nonequilibrium two-phase flows in VFRs.

\subsection{Model equations}

We use the two-phase $(\alpha=o, w)$ extension of Darcy's law for constant fluid densities and Eqs. 2 and 3 for Barenblatt's approach in the matrix system

$$
\begin{gathered}
\mathbf{u}_{\alpha f}^{*}=-\frac{k_{f}^{*} k_{r \alpha, f}}{\mu_{\alpha f}} \nabla\left(P_{\alpha f}+\rho_{\alpha} g z\right):=-\lambda_{\alpha f}^{*} \nabla \Phi_{\alpha f}, \\
\mathbf{u}_{\alpha m}=-\frac{k_{m} k_{r \alpha, m}(\eta)}{\mu_{\alpha m}} \nabla \Phi_{\alpha m}:=-\lambda_{\alpha m}(\eta) \nabla \Phi_{\alpha m} .
\end{gathered}
$$

Again, we assume that non-equilibrium effects in the fracture system are negligible due to the higher permeability and we only consider non-equilibrium effects in the matrix system. Note that for the conventional two-phase flow system of equations and for the nonequilibrium theory by Hassanizadeh, the phase mobility $\lambda_{\alpha}$ in Eq. 7 is a function of water saturation $S_{w}$ instead of the effective water saturation $\eta$. In Eq. 7, the superscript $(*)$ denotes intrinsic (local) fracture properties. The intrinsic fracture permeability evaluated inside the fracture is denoted by $k_{f}^{*}$. We define the intrinsic fracture permeability $k_{f}^{*}$ based on the fracture aperture. The matrix permeability is denoted by $k_{m}$. Relative permeabilities are denoted by $k_{r w, \zeta}$ and $k_{r o, \zeta}$, where $\zeta=f, m$ indicates the fracture or matrix systems. Here $P$ is the pressure, $\rho$ is the fluid density, $g$ is the gravity acceleration factor and $z$ is the vertical upward direction. We define a phase mobility $\lambda_{\alpha}=k \times k_{r \alpha} / \mu_{\alpha}$ as the ratio between the phase permeability and the fluid viscosity. The phase potential $\Phi_{\alpha}$ is equal to the pressure plus the gravity term.

The mass conservation equation reads

$$
\frac{\partial}{\partial t}\left(\varphi_{\zeta} \rho_{\alpha \zeta} S_{\alpha \zeta}\right)+\nabla \cdot\left(\mathbf{u}_{\alpha \zeta} \rho_{\alpha \zeta}\right)=0,
$$


where $\varphi$ is the porosity and $S_{\alpha}$ is the phase saturation. We obtain the governing equations describing nonequilibrium incompressible two-phase flow by combining Darcy's Law (Eq. 7) and mass conversation (Eq. 8)

$$
\begin{aligned}
& \frac{\partial}{\partial t}\left(\varphi_{f}^{*} \rho_{\alpha f} S_{\alpha f}\right)=\nabla \cdot\left(\rho_{\alpha f} \lambda_{\alpha f}^{*} \nabla \Phi_{\alpha f}\right) \text { in } \Omega_{f}, \\
& \frac{\partial}{\partial t}\left(\varphi_{m} \rho_{\alpha m} S_{\alpha m}\right)=\nabla \cdot\left(\rho_{\alpha m} \lambda_{\alpha m}(\eta) \nabla \Phi_{\alpha m}\right) \text { in } \Omega_{m} .
\end{aligned}
$$

At the interface between the fracture and matrix systems, there is continuity of water and oil flow

$$
\left(\rho_{\alpha f} \lambda_{\alpha f}^{*} \nabla \Phi_{\alpha f}\right) \cdot \mathbf{n}=\left(\rho_{\alpha m} \lambda_{\alpha m}(\eta) \nabla \Phi_{\alpha m}\right) \cdot \mathbf{n} \quad \text { on } \partial \Omega_{m}
$$

where $\mathbf{n}$ denotes the outward unit normal vector to the surface $\left(\partial \Omega_{m}\right)$ pointing from the matrix to the fracture. At the interface, there is also continuity of capillary pressure [60]. Pressure continuity and hence capillarypressure continuity is a basic principle (see e.g., [28]). The only question is how to describe the capillary pressure, either by an equilibrium (static) expression or by a non-equilibrium (dynamic) expression. For Barenblatt's approach, the phase-pressure difference is defined as a non-equilibrium capillary pressure that is a function of $\eta$, i.e., $P_{o}-P_{w}=P_{c}(\eta)$. However, for Hassanizadeh's approach, the phase-pressure difference is equal to the dynamic capillary pressure $P_{o}-P_{w}=P_{c}^{d y n}\left(S_{w}\right)$. As mentioned earlier, for the conventional two-phase flow without nonequilibrium effects, we define a capillary pressure $P_{o}-P_{w}=P_{c}^{\text {stat }}\left(S_{w}\right)$. We assume that the capillary pressure in the fracture can be represented by a static expression as (imbibition or drainage) equilibrium is relatively easily established due to the higher permeability, whereas in the low permeability matrix column, we can expect significant delay times to reach capillary equilibrium. Therefore, we equate the fracture capillary pressure to the non-equilibrium matrix capillary pressure at the boundary between them, i.e.,

$P_{c m}\left(t, \mathbf{r}_{b}, \mathbf{r}_{s}, \eta\right)=P_{c f}\left(t, \mathbf{r}_{b}, S_{w f}\right)$ for Barenblatt, $P_{c m}^{d y n}\left(t, \mathbf{r}_{b}, \mathbf{r}_{s}, S_{w}\right)=P_{c f}\left(t, \mathbf{r}_{b}, S_{w f}\right)$ for Hassanizadeh.

In Eq. 12, $\mathbf{r}_{b}$ and $\mathbf{r}_{s}$ denote the large-scale coordinate and the local-(small)-scale coordinate, respectively.

\section{Upscaled model}

We start with two-phase flow equations at the Darcy scale to obtain the upscaled equations including nonequilibrium effects for a VFR at the global scale (gridblock scale) using homogenization theory. The upscaling technique (homogenization) consists of five major steps.

The first step in homogenization is the subdivision of the horizontal coordinates of the vertically fractured reservoir into two scales (see Fig. 1): the local (small units) scale of size $(l)$ and the global scale of size $(L)$ that is much larger than the local scale. This implies that a condition for the application of homogenization is that separation of scales is possible. Our choice for the small-unit (SU) scale is a single matrix column of which the vertical faces are surrounded by fractures and its horizontal faces (e.g., top and bottom) are connected to the cap and base rock, which is warranted for our model (see Fig. 1). Our choice of using a single matrix column is instigated by the incompatibility of larger unit cells (leading to a more accurate determination of effective properties) with a separation of scale. We define $L$ as the dimension of the grid-block scale. Note that each grid-block consists of a few hundred small units. We define a scaling ratio $\varepsilon=$ $l / L$ between the local scale and the global scale. A very large difference between the size of the global scale (grid block) and the local scale (SU) in addition to the low permeability of the matrix columns, suggests that the oil flux from the matrix columns to the fractures only leads to local scale variations of the fracture potential. As a consequence of the separation of scales, any space-dependent quantity required to describe the physical process is a function of these two scales. Therefore, we can split the gradient operator $(\nabla)$ into a global-scale (big) term, $\nabla_{b}$, operating on the large-scale coordinate $\mathbf{r}_{b}$ and term, $\nabla_{s}$, operating on the local-(small)scale coordinate $\mathbf{r}_{s}$ where $\nabla=\nabla_{b}+\nabla_{s}$. The matrix equation acts at the local scale. Thus, we do not apply the splitting procedure to the matrix domain. However, in a vertically fractured reservoir, there is no separation of scales in the vertical direction because the global scale is the same as the local scale. Therefore, we only apply the splitting procedure to the $x$ - and $y$-component of the fracture-gradient operator. 
The second step in the homogenization technique is writing the equations at the local scale in a dimensionless form using characteristic reference quantities. Reference quantities assume values that can be indicated in the problem of interest or are made up by combining the reference quantities. In our model, we use $\ell$ and $L$ as the characteristic lengths for local-scale and global-scale (grid-block scale) differentiation. This results in $\nabla_{D}=\nabla_{b}+$ $\varepsilon^{-1} \nabla_{s}$. The reference potential, $\Phi_{R}$, is the potential difference between the injection and production well $(\Delta \Phi)$. We use $t_{R}=L^{2} \mu /\left(k_{f} \Delta \Phi\right)$ as the reference time for both the fracture and matrix system.

The third step in the homogenization technique is expressing the solution variables in the form of an asymptotic expansion in powers of $\varepsilon$.

The fourth step in the homogenization technique is solving the successive boundary-value problems that are obtained after introducing the asymptotic expansion in the local dimensionless descriptions. We collect the terms that have the same significance as shown by the characteristic power of $\varepsilon$. Different upscaled equations are obtained when dimensionless numbers assume values of different orders of magnitude with respect to $\varepsilon$. We evaluate the permeability ratio with respect to the scaling ratio $\varepsilon$. If one takes the ratio of the two permeabilities $k_{m} / k_{f}^{*}$ to be of order one, then homogenization theory will lead to an upscaled single-porosity model. If the ratio is smaller than $\varepsilon^{2}$, then the matrix blocks do not contribute to the global continuity system of equations in the upscaled model, which then corresponds to the upscaled equation only for the fractures. Hence, we consider that $k_{m} / k_{f}^{*} \sim \varepsilon^{2}$, because this is the regime for which a reservoir is a fractured reservoir from the fluid-flow point of view, i.e., naturally occurring fractures have a significant effect on fluid flow. Again, $k_{f}^{*}$ denotes the intrinsic fracture permeability evaluated inside the fracture. We start by solving the equations of the lowest order in $\varepsilon$.

The fifth step in the homogenization technique is obtaining the upscaled (macroscopic equivalent) model. We define the macroscopic average of any parameter $\mathbf{q}$ over the small unit volume $\Omega$ by integrating over $\Omega_{f}$ and dividing by $|\Omega|$. After solving the boundary-value problems and removing the local-scale $\left(\partial / \partial_{s}\right)$ dependence from the upscaled model by applying an averaging procedure and Gauss's theorem to the boundary condition, the upscaled equations including the non-equilibrium effects of Barenblatt in the VFR with homogenization are

$$
\begin{aligned}
& \frac{\partial}{\partial t}\left(\varphi_{f} S_{w f}\right)-\nabla_{b} \cdot\left(\lambda_{w f} \nabla_{b} \Phi_{w f}\right) \\
& +\frac{1}{|\Omega|} \int_{\Omega_{m}}\left\{\frac{\partial}{\partial t}\left(\varphi_{m} S_{w m}\right)-\frac{\partial}{\partial z}\left(\lambda_{w m}(\eta) \frac{\partial}{\partial z} \Phi_{w m}\right)\right\} \mathrm{d} \mathbf{x}_{s} \\
& =q_{e x t, w} \quad \text { in } Q \\
& -\nabla_{b} \cdot\left(\left(\lambda_{w f}+\lambda_{o f}\right) \nabla_{b} \Phi_{w f}+\lambda_{o f} \nabla_{b} \Phi_{c f}\right) \\
& +\frac{1}{|\Omega|} \int_{\Omega_{m}}\left\{-\frac{\partial}{\partial z}\left(\left(\lambda_{w m}(\eta)+\lambda_{o m}(\eta)\right) \frac{\partial}{\partial z} \Phi_{w m}\right.\right. \\
& \left.\left.\quad+\lambda_{o m}(\eta) \frac{\partial}{\partial z} \Phi_{c m}(\eta)\right)\right\} \mathrm{d} \mathbf{x}_{s}= \\
& =q_{e x t, w}+q_{e x t, o} \quad \text { in } Q,
\end{aligned}
$$

where $q_{\text {ext,w }}$ and $q_{\text {ext,o }}$ are the external flow rates, which come from the production and injection wells. The phase potential $\Phi_{\alpha}$ is equal to the pressure $(P)$ plus the gravity term. Here, we also define the non-equilibrium capillary potential $\Phi_{c}(\eta)=$ $P_{c}(\eta)+\left(\rho_{o}-\rho_{w}\right) g z$. The integral term is the exchange term of fluid flow at the interface between the fracture and matrix. Here, the global fracture porosity and (effective) permeability are given as

$$
\begin{aligned}
& \varphi_{f}=\frac{1}{|\Omega|} \int_{\Omega_{f}} \varphi_{f}^{*} \mathrm{~d} \mathbf{x}_{s}, \\
& k_{f}=\frac{1}{|\Omega|} \int_{\Omega_{f}} k_{f}^{*}\left(\mathrm{I}+\nabla_{s} \otimes\left(\omega_{1}, \omega_{2}, 0\right)\right) \mathrm{d} \mathbf{x}_{s} .
\end{aligned}
$$

We use $\varphi_{f}^{*}$ to denote the intrinsic fracture porosity, and $|\Omega|$ to denote the combined fracture and matrix domain. The integration is over the local domain, which is a small unit cell that contains a specific matrixcolumn size and fracture aperture for the grid cell. In the same way, we use $k_{f}^{*}$ to denote the intrinsic fracture permeability. The behavior of $\omega_{1}\left(\omega_{2}\right)$ is a measure of the potential fluctuation caused by the nonhomogeneous nature of the fractured reservoir that is subjected to a global potential gradient in the $x$ direction ( $y$-direction). The VFR does not need an upscaling procedure in the $z$-direction, which is a reason that we only consider the vector $\left(\omega_{1}, \omega_{2}, 0\right)$. The cell equation required to solve for $\left(\omega_{1}, \omega_{2}, 0\right)$ can be found in Salimi and Bruining [45]. 
The upscaled equations including the non-equilibrium effects of Hassanizadeh in the VFR read

$$
\begin{aligned}
& \frac{\partial}{\partial t}\left(\varphi_{f} S_{w f}\right)-\nabla_{b} \cdot\left(\lambda_{w f} \nabla_{b} \Phi_{w f}\right)+ \\
& +\frac{1}{|\Omega|} \int_{\Omega_{m}}\left\{\frac{\partial}{\partial t}\left(\varphi_{m} S_{w m}\right)-\frac{\partial}{\partial z}\left(\lambda_{w m}\left(S_{w m}\right) \frac{\partial}{\partial z} \Phi_{w m}\right)\right\} \mathrm{d} \mathbf{x}_{s} \\
& =q_{\text {ext }, w} \text { in } Q \\
& -\nabla_{b} \cdot\left(\left(\lambda_{w f}+\lambda_{o f}\right) \nabla_{b} \Phi_{w f}+\lambda_{o f} \nabla_{b} \Phi_{c f}\right)+ \\
& +\frac{1}{|\Omega|} \int_{\Omega_{m}}\left\{-\frac{\partial}{\partial z}\left(\left(\lambda_{w m}\left(S_{w m}\right)+\lambda_{o m}\left(S_{w m}\right)\right) \frac{\partial}{\partial z} \Phi_{w m}\right.\right. \\
& \left.\left.\quad+\lambda_{o m}\left(S_{w m}\right) \frac{\partial}{\partial z} \Phi_{c m}^{d y n}\right)\right\} \mathrm{d} \mathbf{x}_{s} \\
& =q_{\text {ext }, w}+q_{\text {ext }, o} \quad \text { in } Q,
\end{aligned}
$$

where now the phase mobilities $\lambda_{\alpha m}$ in Eqs. 17 and 18 are a function of the water saturation $S_{w}$ and not of the effective water saturation $\eta$ as in Eqs. 13 and 14. Moreover, the matrix capillary potential $\Phi_{c m}(\eta)$ in Eq. 14 is replaced by $\Phi_{c m}^{d y n}$ in Eq. 18.

For the non-equilibrium theory by Barenblatt, the local problem for the effective saturation $\eta$, real saturation $S_{w}$, and potential $\Phi_{w m}$ on the matrix column at the point $\mathbf{r}_{b}$ results from the $\varepsilon^{0}$ terms of Eqs. 3, 10 and 12. The equations for the matrix columns using Barenblatt's approach are

$$
\begin{aligned}
& \frac{\partial}{\partial t}\left(\varphi_{m} S_{w m}\right)-\nabla_{s} \cdot\left(\lambda_{w m}(\eta) \nabla_{s} \Phi_{w m}\right)=0 \quad \text { in } B_{m} \\
& -\nabla_{s} \cdot\left(\left(\lambda_{w m}(\eta)+\lambda_{o m}(\eta)\right) \nabla_{s} \Phi_{w m}\right. \\
& \left.\quad+\lambda_{o m}(\eta) \nabla_{s} \Phi_{c m}(\eta)\right)=0 \text { in } B_{m}
\end{aligned}
$$

$\tau_{b} \frac{\partial S_{w}}{\partial t}-\eta+S_{w}=0$

The superscript (0) is dropped for reasons of concise notation. At the interface between the fracture and matrix systems, there is continuity of water and oil flow and continuity of capillary pressure. The boundary conditions on the vertical faces of the matrix columns read

$$
\begin{aligned}
& \Phi_{w m}\left(t, \mathbf{r}_{b}, \mathbf{r}_{s}\right)=\Phi_{w f}\left(t, \mathbf{r}_{b}\right), \quad \text { and } \\
& \Phi_{c m}\left(t, \mathbf{r}_{b}, \mathbf{r}_{s}, \eta\right)=\Phi_{c f}\left(t, \mathbf{r}_{b}\right),
\end{aligned}
$$

where $\mathbf{r}_{b}$ denotes the global scale and $\mathbf{r}_{s}$ denotes the local scale.

For the non-equilibrium theory by Hassanizadeh et al., the local equations for the real saturation, dynamic capillary pressure, and potential on the matrix column at the point $\mathbf{r}_{b}$ results from the $\varepsilon^{0}$ terms of Eqs. 4, 10 and 12; they read

$$
\frac{\partial}{\partial t}\left(\varphi_{m} S_{w m}\right)-\nabla_{s} \cdot\left(\lambda_{w m}\left(S_{w m}\right) \nabla_{s} \Phi_{w m}\right)=0 \text { in } B_{m},
$$

$$
\begin{aligned}
-\nabla_{s} \cdot & \left(\left(\lambda_{w m}\left(S_{w m}\right)+\lambda_{o m}\left(S_{w m}\right)\right) \nabla_{s} \Phi_{w m}\right. \\
& \left.+\lambda_{o m}\left(S_{w m}\right) \nabla_{s} \Phi_{c m}^{d y n}\right)=0 \text { in } B_{m},
\end{aligned}
$$

$\tau_{h} \frac{\partial S_{w}}{\partial t}+P_{c}^{d y n}-P_{c}^{s t a t}=0 \quad$ in $B_{m}$,

$\Phi_{w m}\left(t, \mathbf{r}_{b}, \mathbf{r}_{s}\right)=\Phi_{w f}\left(t, \mathbf{r}_{b}\right), \quad$ and

$\Phi_{c m}^{d y n}\left(t, \mathbf{r}_{b}, \mathbf{r}_{s}\right)=\Phi_{c f}\left(t, \mathbf{r}_{b}\right) \quad$ on $\partial B_{m}$

There are no-flow boundary conditions on the top and bottom of the entire fractured reservoir, i.e.,

$-\lambda_{\alpha \zeta} \nabla \Phi_{\alpha \zeta} \cdot \mathbf{n}=0, \quad \alpha=w, o, \quad$ and $\quad \zeta=f, m$,

where $\mathbf{n}$ denotes the outward unit normal vector to the surface.

Our choice for the small-unit scale is a single matrix column of which the vertical faces are surrounded by fractures and the horizontal faces (e.g., top and bottom) are connected to the cap and base rock. We define the global scale as the dimension of the grid-block scale.

The derivation of the upscaled model including the non-equilibrium effects is complete. In the next section, we develop an efficient numerical method to solve the complex system of equations in a vertically fractured reservoir.

\section{Numerical solution}

The numerical procedure described below is an extension of the method used by Arbogast [5] and Salimi and Bruining $[42,44]$ for the sugar-cube model. The difficulty arises due to the coupling of flow in the 
vertical direction, which is important in the upscaledVFR model. From the computational point of view, we consider a matrix column associated with each point in the base plane of the reservoir. The horizontal crosssectional position of any point $r_{b}=\left(x_{b}, y_{b}, z_{b}\right) \in Q$, is denoted by $r_{b}^{\prime}=\left(x_{b}, y_{b}\right) \in A_{Q}$. The matrix column at $r_{b}^{\prime}=\left(x_{b}, y_{b}\right) \in A_{Q}$ is denoted by $B_{m}\left(r_{b}^{\prime}\right)=\Omega_{m}\left(r_{b}^{\prime}\right) \times$ $H$, where this matrix column is representative of the matrix columns in the vicinity of $r^{\prime}$. We formulate our numerical method in terms of the water potential, the capillary pressure, and the water saturation. Here, we also define the capillary potential $\Phi_{c}(\eta)=P_{c}(\eta)+$ $\left(\rho_{o}-\rho_{w}\right) g z$. We assume that all external sources, i.e., the production and injection wells, influence only the fractures. Equations 17, 19, and 23 above are called the saturation equations, and the sums over the phases of each of the two-phase equations are Eqs. 18, 20 and 24, the pressure equations.

Initially, there is capillary-gravity equilibrium both in the fracture system and in the matrix column. This means that the fluid-exchange term between fracture and matrix is zero initially. In addition, the effective water saturation $\eta$ is equal to the actual water saturation $S_{w}$ for Barenblatt's approach, and the dynamic capillary potential $\Phi_{c m}^{d y n}$ is the same as the static (equilibrium) capillary potential $\Phi_{c m}^{\text {stat }}$ for Hassanizadeh's approach. In other words, in the non-equilibrium theory by Hassanizadeh, there is continuity of dynamic capillary pressure, i.e., between the fracture capillary potential and the matrix dynamic capillary potential instead of continuity of the static capillary potential. Since the initial oil-in-place is known, we determine the initial fracture water potential by solving the fracture pressure equation (Eq. 18). Because of equilibrium, we can solve the matrix pressure equation (Eq. 20 for Barenblatt and Eq. 24 for Hassanizadeh) to obtain the initial matrix water potential.

Equations 17-27 cannot be solved sequentially or explicitly, because a small change in the boundary values on each matrix column can cause flow of a volume of fluid that is large in comparison to the volume of the fractures [13]. In other words, the matrix absorbs more fluid from the surrounding fractures in one step than can be resident there. Part of the excess volume in the matrix is returned to the fractures in the next step, however, violating mass conservation. Therefore, the fracture-matrix interaction must be handled implicitly.

We use a backward-Euler time approximation for the complete system of Eqs. 17-27. We further use a finite-volume approach and first-order upwind scheme for spatial discretization. To facilitate the implementation of the no-flow boundary conditions and the continuity conditions of the potentials along the fracture- matrix interfaces, we discretize the space variables in a cell-centered manner in the fractures and also cellcentered with respect to $z$ in the matrix columns. However, the discretization in the $x_{s}$ and $y_{s}$ are vertexcentered. The reason to use vertex-centered cells in the $x$ and $y$ direction is its convenience for Dirichlet boundary conditions at the interface between fracture and matrix, i.e., continuity of the phase potential and capillary pressure. We have not encountered mass balance errors, because the matrix and fracturesystem of equations have been coupled and solved simultaneously implicitly. The simultaneous implicit approach avoids that a small change in the boundary values on each matrix column can cause flow of a volume of fluid that is larger than the volume of the fractures. In this work, we use uniform grid cells in the fractures and in each matrix column. From the computational perspective, we consider a case in which the vertical discretization in the matrix columns coincides with that in the fractures.

We discretize $Q$ into $N_{x f} \times N_{y f} \times N_{z f}$ grid cells. After that, we can write the fully discrete, nonlinear fracture and matrix equations

$$
\left\{\begin{array}{l}
F_{i}\left(\vec{\Phi}_{w f}^{n}, \vec{S}_{w f}^{n}, \vec{\Phi}_{w m}^{n}, \vec{S}_{w m}^{n}\right)=0 \\
\quad i=1,2, \ldots, N_{x f} \times N_{y f} \times N_{z f}, \\
M_{i^{\prime} j}\left(\vec{\Phi}_{w f}^{n}, \vec{S}_{w f}^{n}, \vec{\Phi}_{w m, i^{\prime}}^{n}, \vec{S}_{w m, i^{\prime}}^{n}, \vec{\eta}_{w m, i^{\prime}}^{n}\right)=0 \\
\quad\left\{\begin{array}{l}
i^{\prime}=1,2, \ldots, N_{x f} \times N_{y f}, \\
j=1,2, \ldots, N_{x m} \times N_{y m} \times N_{z f},
\end{array}\right.
\end{array}\right.
$$

where $F_{i}$ and $M_{i^{\prime} j}$ are nonlinear functions, which represent the discretized version of the model equations (Eqs. 17-27). For Hassanizadeh's approach, the unknown vector of the effective water saturation $\vec{\eta}_{w m, i^{\prime}}^{n}$ is replaced by an unknown vector of the dynamic capillary potential $\Phi_{c m}^{d y n}$. We use Newton's method to solve the above system of equations. The conventional Newton procedure leads to an efficient numerical scheme. Such a conventional procedure would run as follows:

(1) Start with an initial guess for the solution

$$
\vec{\Phi}_{w f}^{n, 0}, \vec{S}_{w f}^{n, 0}, \vec{\Phi}_{w m}^{n, 0}, \vec{S}_{w m}^{n, 0} \text {, and } \vec{\eta}_{w m}^{n, 0} .
$$

(2) For each $k=1,2, \ldots, \kappa$ until convergence is reached:

a. Solve for the $n$th time level's solution

$$
\vec{\Phi}_{w f}^{n, k}, \vec{S}_{w f}^{n, k}, \vec{\Phi}_{w m}^{n, k}, \vec{S}_{w m}^{n, k} \text {, and } \vec{\eta}_{w m}^{n, k},
$$


satisfying

$$
\begin{aligned}
& \int F_{i}^{n, k-1}+\sum_{i^{\prime \prime}=1}^{N_{f}}\left\{\left[\partial_{\Phi_{w f, i^{\prime}}} F_{i}^{n, k-1} \delta \Phi_{w f, i^{\prime \prime}}^{n, k}+\partial S_{w f, i^{\prime \prime}} F_{i}^{n, k-1} \delta S_{w f, i^{\prime \prime}}^{n, k}\right]\right. \\
& \left.+\sum_{j^{\prime}=1}^{N_{m}}\left[\begin{array}{c}
\partial_{\Phi_{w m, i^{\prime \prime} j^{\prime}}} F_{i}^{n, k-1} \delta \Phi_{w m, i^{\prime \prime} j^{\prime}}^{n, k} \\
+\partial_{S_{w m, i^{\prime \prime} j^{\prime}}} F_{i}^{n, k-1} \delta S_{w m, i^{\prime \prime} j^{\prime}}^{n, k} \\
+\partial_{\eta_{w m, i^{\prime} j^{\prime} j^{\prime}} F_{i}^{n, k-1} \delta \eta_{w m, i^{\prime \prime} j^{\prime}}^{n, k}}
\end{array}\right]\right\}=0, \\
& i=1,2, \ldots, N_{f} \text {, } \\
& M_{i^{\prime} j}^{n, k-1}+\sum_{l=1}^{N_{z f}}\left[\begin{array}{l}
\partial_{\Phi_{w f,\left(i^{\prime}, l\right)}} M_{i^{\prime} j}^{n, k-1} \delta \Phi_{w f,\left(i^{\prime}, l\right)}^{n, k} \\
+\partial_{w f,\left(i^{\prime}, l\right)} M_{i^{\prime} j}^{n, k-1} \delta S_{w f,\left(i^{\prime}, l\right)}^{n, k}
\end{array}\right] \\
& \left\{\begin{array}{l}
i^{\prime}=1,2, \ldots, N_{x f} \times N_{y f}, \\
j=1,2, \ldots, N_{m} .
\end{array}\right. \\
& +\sum_{j^{\prime}=1}^{N_{m}}\left[\begin{array}{c}
\partial_{\Phi_{w m, i^{\prime} j^{\prime}}} M_{i^{\prime} j}^{n, k-1} \delta \Phi_{w m, i^{\prime} j^{\prime}}^{n, k} \\
+\partial_{S_{w m, i^{\prime} j^{\prime}}} M_{i^{\prime} j}^{n, k-1} \delta S_{w m, i^{\prime} j^{\prime}}^{n, k} \\
+\partial_{\eta_{w m, i^{\prime} j^{\prime}} M_{i^{\prime} j}^{n, k-1} \delta \eta_{w m, i^{\prime} j^{\prime}}^{n, k}}
\end{array}\right]=0,
\end{aligned}
$$

Here $\partial_{\pi}$ denotes the partial derivative with respect to $\pi$.

b. Update the potentials and saturations

$$
\begin{aligned}
& \vec{\Phi}_{w f}^{n, k}=\vec{\Phi}_{w f}^{n, k-1}+\delta \vec{\Phi}_{w f}^{n, k}, \quad \vec{S}_{w f}^{n, k}=\vec{S}_{w f}^{n, k-1}+\delta \vec{S}_{w f}^{n, k}, \\
& \vec{\Phi}_{w m}^{n, k}=\vec{\Phi}_{w m}^{n, k-1}+\delta \vec{\Phi}_{w m}^{n, k}, \quad \vec{S}_{w m}^{n, k}=\vec{S}_{w m}^{n, k-1}+\delta \vec{S}_{w m}^{n, k}, \\
& \vec{\eta}_{w m}^{n, k}=\vec{\eta}_{w m}^{n, k-1}+\delta \vec{\eta}_{w m}^{n, k} .
\end{aligned}
$$

This will be continued for $k=1, \ldots \kappa$, i.e., until convergence is obtained. This would complete the algorithm for a time step. The above linear system (Eq. 29) involves the solution of a $\left(2 \times N_{f}+3 \times N_{x f} \times N_{y f} \times\right.$ $\left.N_{m}\right) \times\left(2 \times N_{f}+3 \times N_{x f} \times N_{y f} \times N_{m}\right)$ matrix for each Newton iteration at a time step, which is computationally expensive. Within the linearized Newton problem (Eq. 29), the matrix solutions in the $i^{\prime}$ th column depend on $\Phi_{w f,\left(i^{\prime}, l\right)}^{n, k}$ and $S_{w f,\left(i^{\prime}, l\right)}^{n, k}$, where $l=1,2, \ldots, N_{z f}$. In other words, the matrix solution in the $i^{\prime}$ th column only depends locally on all the fracture cells surrounding the matrix column of interest. It is therefore possible to develop an efficient numerical scheme by decoupling the matrix and fracture problems without affecting the implicit nature of the scheme. We replace the matrix problem in Eq. 29 by the following three types of problems for

$\left(\tilde{\delta} \vec{\Phi}_{w m,\left(i^{\prime}, l\right)}^{n, m}, \tilde{\delta} \vec{S}_{w m,\left(i^{\prime}, l\right)}^{n, m}, \tilde{\delta} \vec{\eta}_{w m,\left(i^{\prime}, l\right)}^{n, m}\right)$, $\left(\hat{\delta} \vec{\Phi}_{w m,\left(i^{\prime}, l\right)}^{n, m}, \hat{\delta} \vec{S}_{w m,\left(i^{\prime}, l\right)}^{n, m}, \tilde{\delta} \vec{\eta}_{w m,\left(i^{\prime}, l\right)}^{n, m}\right)$, and $\left(\bar{\delta} \vec{\Phi}_{w m, i^{\prime}}^{n, m}, \bar{\delta} \vec{S}_{w m, i^{\prime}}^{n, m}, \tilde{\delta} \vec{\eta}_{w m, i^{\prime}}^{n, m}\right)$, where $\tilde{\delta}$ 's, $\hat{\delta}$ 's, and $\bar{\delta}$ 's satisfy three types of problems as follows:

Firstly, for each $i^{\prime}=1,2, \ldots, N_{x f} \times N_{y f}$ and $j=1,2$, $3, \ldots, N_{m}$

$$
M_{i^{\prime} j}^{n, k-1}+\sum_{j^{\prime}=1}^{N_{m}}\left[\begin{array}{c}
\partial_{\Phi_{w m, i^{\prime} j^{\prime}}} M_{i^{\prime} j}^{n, k-1} \bar{\delta} \Phi_{w m, i^{\prime} j^{\prime}}^{n, k} \\
+\partial_{S_{w m, i^{\prime} j^{\prime}}} M_{i^{\prime} j}^{n, k-1} \bar{\delta} S_{w m, i^{\prime} j^{\prime}}^{n, k} \\
+\partial_{\eta_{w m, i^{\prime} j^{\prime}}} M_{i^{\prime} j}^{n, k-1} \bar{\delta} \eta_{w m, i^{\prime} j^{\prime}}^{n, k}
\end{array}\right]=0,
$$

secondly, for $l=1,2, \ldots, N_{z f}$,

$\partial_{\Phi_{w f,\left(i^{\prime}, l\right)}} M_{i^{\prime} j}^{n, k-1}+\sum_{j^{\prime}=1}^{N_{m}}\left[\begin{array}{c}\partial_{\Phi_{w m, l^{\prime} j^{\prime}}} M_{i^{\prime} j}^{n, k-1} \tilde{\delta} \Phi_{w m,\left(i^{\prime}, l\right), j^{\prime}}^{n, k} \\ +\partial_{S_{w m, i^{\prime} j^{\prime}}} M_{i^{\prime} j}^{n, k-1} \tilde{\delta} S_{w m,\left(i^{\prime}, l\right), j^{\prime}}^{n, k} \\ +\partial_{\eta_{w m, i^{\prime} j^{\prime}}} M_{i^{\prime} j}^{n, k-1} \tilde{\delta} \eta_{w m,\left(i^{\prime}, l\right), j^{\prime}}^{n, k}\end{array}\right]=0$,

thirdly, for $l=1,2, \ldots, N_{z f}$,

$\partial_{S_{\left.w f, i^{\prime}, l\right)}} M_{i^{\prime} j}^{n, k-1}+\sum_{j^{\prime}=1}^{N_{m}}\left[\begin{array}{c}\partial_{\Phi_{w m, i^{\prime} j^{\prime}}} M_{i^{\prime} j}^{n, k-1} \hat{\delta} \Phi_{w m,\left(i^{\prime}, l\right), j^{\prime}}^{n, k} \\ +\partial_{S_{w m, i^{\prime} j^{\prime}}} M_{i^{\prime} j}^{n, k-1} \hat{\delta} S_{w m,\left(i^{\prime}, l\right), j^{\prime}}^{n, k} \\ +\partial_{\eta_{w m, i^{\prime} j^{\prime}}} M_{i^{\prime} j}^{n, k-1} \hat{\delta} \eta_{w m,\left(i^{\prime}, l\right), j^{\prime}}^{n, k}\end{array}\right]=0$.

If we multiply Eq. 31 by $\delta \Phi_{w f,\left(i^{\prime}, l\right)}^{n, k}$ and Eq. 32 by $\delta S_{w f,\left(i^{\prime}, l\right)}^{n, k}$ and then add these equations to Eq. 30, the result would be identical to the matrix problem in Eq. 29. As a result, the matrix unknowns can be calculated by

$$
\begin{aligned}
\delta \Phi_{w m, i^{\prime} j}^{n, k}=\bar{\delta} \Phi_{w m, i^{\prime} j}^{n, k}+\sum_{l=1}^{N_{z f}}\left(\tilde{\delta} \Phi_{w m,\left(i^{\prime}, l\right), j}^{n, k} \delta \Phi_{w f,\left(i^{\prime}, l\right)}^{n, k}\right. & \\
& \left.+\hat{\delta} \Phi_{w m,\left(i^{\prime}, l\right), j}^{n, k} \delta S_{w f,\left(i^{\prime}, l\right)}^{n, k}\right)
\end{aligned}
$$

$$
\begin{aligned}
\delta S_{w m, i^{\prime} j}^{n, k}=\bar{\delta} S_{w m, i^{\prime} j}^{n, k}+\sum_{l=1}^{N_{z f}}(\tilde{\delta} & S_{w m,\left(i^{\prime}, l\right), j}^{n, k} \delta \Phi_{w f,\left(i^{\prime}, l\right)}^{n, k} \\
& \left.+\hat{\delta} S_{w m,\left(i^{\prime}, l\right), j}^{n, k} \delta S_{w f,\left(i^{\prime}, l\right)}^{n, k}\right),
\end{aligned}
$$

$$
\begin{aligned}
\delta \eta_{w m, i^{\prime} j}^{n, k}=\bar{\delta} \eta_{w m, i^{\prime} j}^{n, k}+\sum_{l=1}^{N_{z f}}(\tilde{\delta} & \eta_{w m,\left(i^{\prime}, l\right), j}^{n, k} \delta \Phi_{w f,\left(i^{\prime}, l\right)}^{n, k} \\
& \left.+\hat{\delta} \eta_{w m,\left(i^{\prime}, l\right), j}^{n, k} \delta S_{w f,\left(i^{\prime}, l\right)}^{n, k}\right)
\end{aligned}
$$

Equations 30-32 can be solved independently of the fracture system. Thus, we modify step 2a of the Newton Algorithm by first solving Eqs. 30-32. The changes in the fracture unknowns are then given by solving the fracture equations of Eq. 29, using implicitly definition of Eqs. 33-35. Subsequently, we explicitly use the changes in the fracture unknowns and Eqs. 30-32 to 
update the matrix $\delta$-potential (Eq. 33) and matrix $\delta$ saturations (Eqs. 34 and 35). Finally, the Newton iteration can be continued. This efficient numerical method is inexpensive as it only involves the solution of many $\left(3 \times N_{m}\right) \times\left(3 \times N_{m}\right)$ matrices and the solution of a $\left(2 \times N_{f}\right) \times\left(2 \times N_{f}\right)$ matrix as opposed to a single big matrix.

A similar procedure can be given in order to develop an efficient numerical scheme for solving the upscaled equation including the non-equilibrium capillary potential by Hassanizadeh's approach. To do this, we replace the matrix effective water saturation $\eta_{w m}$ everywhere it appears in Eqs. 28-35 by the dynamic capillary potential $\Phi_{c m}^{d y n}$.

\subsection{Numerical discretization}

We discretize the fractured reservoir into $10 \times 5 \times 9$ grid cells in the $x$-, $y$-, and $z$-direction. Hence, there are 450 grid cells for the fracture system, which contains $10 \times 5$ rock matrix columns, assigned to the baseplane coordinates. Each column, which extends from the base rock to the cap rock, is subdivided in a stack of nine matrix blocks. In other words, corresponding to each fracture grid cell on the base plane, there is a single matrix column, consisting of a stack of nine matrix blocks. We use $9 \times 9 \times 9$ grid cells in the $x$-, $y$-, and $z$-direction for each representative matrix column. Therefore, we have a total of $10 \times 5 \times 9$ grid cells for the fracture part and $(10 \times 5 \times 9) \times 9 \times 9$ grid cells to represent the matrix part, leading to a total of 36,900 grid blocks.

\subsection{Fine-grid model}

To demonstrate the capability of the upscaled-VFR model, we develop a fine-grid model, and compare the results of the fine-grid model with the results of the upscaled-VFR model.

For the fine-grid model, Eqs. 3, 4, 9 and 10 together with the boundary conditions are numerically integrated using a finite-volume approach and first-order upwind scheme with a non-uniform cubic-grid system for spatial discretization. We further use a backwardEuler time approximation for the complete system of equations. The discretization of Eqs. 3, 4, 9 and 10 gives rise to large sparse systems of linear equations. The matrices are ill conditioned because of (1) spatial discontinuity in the permeability of the matrix and fractures, and (2) the small fracture thickness in comparison to the matrix size. Thus, iterative methods may not be suitable unless special robust preconditioners are used [17]. For such problems, direct methods are commonly used in reservoir simulation. In this work, the direct method of D4 Gaussian Elimination is used. Details can be found in Price and Coats [37] and Stringer et al. [55].

The fine-grid model (discrete fracture), which considers the fractures explicitly, includes 2,706,309 cells (681,309 cells for the fractures and 2,025,000 cells for the matrix system). The fine-grid model is a model that includes the fractures explicitly and hence is not an internal check of the validity of the numerical model.

\section{Results and discussion}

This Section presents the results of the numerical simulation. It is important to note that all our results are only valid for truly fractured reservoirs, i.e., reservoirs for which the upscaled fracture permeability is one order of magnitude larger (with respect to the scaling ratio) than the matrix permeability.

\subsection{Simulation parameters}

We consider a vertically fractured oil reservoir with a length of $500 \mathrm{~m}$, width of $200 \mathrm{~m}$, and height of $30 \mathrm{~m}$. Initially, the reservoir is saturated with oil. The initial water saturation both in the fracture and in the matrix is equal to the irreducible water saturations $\left(S_{w, i r}\right)$. As shown in Fig. 3, water is injected through the entire height of the reservoir from one corner and subsequently oil and water are produced through the upper third of the reservoir height at the diagonally opposite corner. Table 1 shows the basic input data for the numerical simulations. For each simulation case, the water injection rate is uniform. Table 2 shows the calculated global (effective) fracture permeabilities based on the different values of the lateral matrix-column size, using Eq. 16. The parameters shown in Table 1 are representative for fractured reservoirs. It can be validated that the parameters correspond to a fractured reservoir, i.e., the main flow is carried by the fractures. This is indeed the case; the intrinsic fracture permeability $k_{f}^{*}$ is $844 \mathrm{D}$, whereas the matrix permeability $k_{m}$ is $1 \mathrm{mD}$.

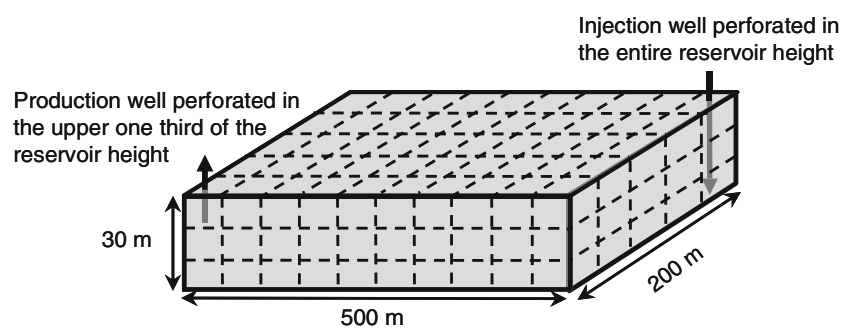

Fig. 3 Fractured reservoir waterflooding pattern 
Table 1 Data used in the numerical simulations

\begin{tabular}{|c|c|c|c|}
\hline Initial reservoir pressure $(\mathrm{MPa})$ & 27.5 & Oil viscosity, $\mu_{o}(\mathrm{cp})$ & 2 \\
\hline $\begin{array}{l}\text { Bottomhole pressure } \\
\text { in production wells }(\mathrm{MPa})\end{array}$ & 26.9 & Oil density, $\rho_{o}\left(\mathrm{~kg} / \mathrm{m}^{3}\right)$ & 833 \\
\hline Well radius $(\mathrm{m})$ & 0.1524 & Water viscosity, $\mu_{w}(\mathrm{cp})$ & 0.5 \\
\hline Fracture aperture, $\delta(\mu \mathrm{m})$ & 100 & Water density, $\rho_{w}\left(\mathrm{~kg} / \mathrm{m}^{3}\right)$ & 1,025 \\
\hline Local fracture porosity, $\varphi_{f}^{*}$ & 1 & Residual oil saturation in matrix, $S_{o r, m}$ & 0.3 \\
\hline $\begin{array}{l}\text { Intrinsic fracture } \\
\quad \text { permeability, } k_{f}^{*}(\mathrm{D})\end{array}$ & 844 & Residual oil saturation in fracture, $S_{o r, f}$ & 0 \\
\hline Matrix porosity, $\varphi_{m}$ & 0.19 & Irreducible water saturation in matrix, $S_{w, i r, m}$ & 0.25 \\
\hline Matrix permeability, $k_{m}(\mathrm{mD})$ & 1 & Irreducible water saturation in fracture, $S_{w, i r, f}$ & 0 \\
\hline
\end{tabular}

This is valid for our upscaled model as $k_{m}<k_{f}<<$ $k_{f}^{*}$. Because fracture and matrix are considered as two different media, we use two different capillary-pressure and relative-permeability curves. Figure 4 shows the capillary-pressure curves for the base case, i.e., contact angle of zero and completely water-wet. Figure 5 shows the relative-permeability curves both for the fracture and for the matrix.

\subsection{Dimensionless numbers}

We characterize the behavior of a VFR by considering the two most important dimensionless numbers. These dimensionless numbers are previously discussed in Salimi and Bruining [45, 47], but for reasons of easy reference, we briefly include the definitions also in this paper. First, we define the Peclet number as the ratio of the capillary-diffusion time in the matrix columns to the residence time of the water in the fracture system. The Peclet number refers to the average flow rate in the fracture system. The local velocity shows large fluctuations. This Peclet number can be expressed as

$P e=\frac{\ell^{2} u_{f w}}{D_{c a p} L}, \quad$ where $\quad D_{c a p}=-\frac{\lambda_{o} \lambda_{w}}{\lambda_{o}+\lambda_{w}} \frac{d P_{c}}{d S_{w}}$.

Here, $\ell$ is the lateral matrix-column size, $u_{f w}$ is the Darcy velocity of water in the fracture, $\lambda_{\alpha}$ is the mobility of phase $\alpha$ (oil, water), and $L$ is the distance between wells. The capillary-diffusion coefficient $D_{\text {cap }}$ is evaluated in the matrix domain. In Eq. 36, the capillary-diffusion coefficient is a strongly nonlinear function of the water saturation. Consequently, its values change with time and space. As mentioned above, the initial water saturations both in the fracture and in the matrix are equal to the irreducible water saturations $\left(S_{w, i r}\right)$. Consequently, the capillary-diffusion coefficient $\left(D_{\text {cap }}\right.$ in Eq. 36) is initially zero for both the fracture and matrix, and remains zero at an early time for those regions that are far from the injection well. Therefore, if we use the geometric mean and/or harmonic mean, the space averaged capillary-diffusion coefficient would become zero. To avoid a zero average capillary-diffusion coefficient, we use an arithmetic average. However, for the average over time, all values are larger than zero and a zero geometric average does not occur. In addition, the geometric mean is a type of mean or average, which indicates the central tendency or typical value of a set of numbers, whereas e.g., the arithmetic mean is not such a robust statistic, meaning that it is greatly influenced by outliers. This is the case for higher injection rates, because the injected water slightly penetrates into the matrix even after long simulation times. In summary, we use an arithmetic mean over space and a geometric mean over time to obtain a single averaged value of the Peclet number for each case discussed below. The second dimensionless number is the gravity number that expresses gravity forces over viscous forces in the fracture system. We use the following expression for this gravity number [50,62]

$N_{G}=\frac{k_{f} \Delta \rho g H}{\mu_{w} u_{f w} L}$,

where $H$ is the height of the reservoir, $k_{f}$ is the effective (global) fracture permeability, $u_{f w}$ is the Darcy velocity of water in the fracture, and $\Delta \rho$ is the density difference between the water and the oil phase.
Table 2 Calculated global fracture permeability and porosity based on lateral matrix-column sizes

\begin{tabular}{|c|c|c|c|}
\hline $\begin{array}{l}\text { Lateral matrix-column } \\
\text { size } \ell(\mathrm{m})\end{array}$ & $\begin{array}{l}\text { Global fracture } \\
\text { permeability in the } x \text { - and } \\
y \text {-direction, } k_{f, x y}(\mathrm{mD})\end{array}$ & $\begin{array}{l}\text { Global fracture } \\
\text { permeability in the } \\
z \text {-direction, } k_{f, z}(\mathrm{mD})\end{array}$ & $\begin{array}{l}\text { Global fracture } \\
\text { porosity, } \varphi_{f}\end{array}$ \\
\hline$\overline{0.5}$ & 169 & 338 & $4 \times 10^{-4}$ \\
\hline 2 & 42 & 84 & $1 \times 10^{-4}$ \\
\hline 4 & 21 & 42 & $5 \times 10^{-5}$ \\
\hline
\end{tabular}




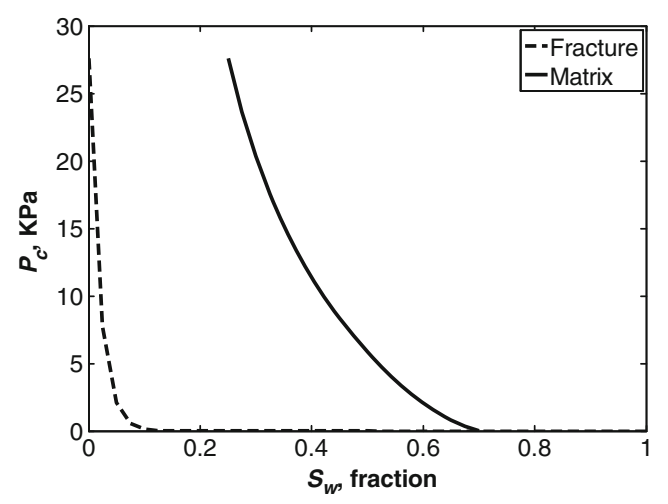

Fig. 4 Capillary-pressure curves of matrix and fracture versus water saturation

\subsection{Comparison between the fine-grid model and the upscaled-VFR model}

Here, we give an example where we compared the results of the fine-grid model with the results of the upscaled-VFR model in which the non-equilibrium effects are included. We assume that the reservoir consists of identical matrix columns of permeability $k_{m}=$ $1 \mathrm{mD}$ and lateral size $\ell=2 \mathrm{~m}$. Moreover, the fractures of the VFR configuration are assumed to have the same aperture. The input data for the fine-grid simulations are summarized in Table 1 (see also Figs. 4 and 5).

Figure $6 \mathrm{a}$ and $\mathrm{b}$ compare the cumulative oil production obtained with the upscaled model with the cumulative oil production obtained with the fine-grid model for a delay time of $\tau_{b}=3 \times 10^{6} \mathrm{~s}$ and a capillarydamping coefficient of $\tau_{h}=1.8 \times 10^{11} \mathrm{~kg} /(\mathrm{m} \mathrm{s})$, respectively. The water injection rate for the results shown in Fig. 6a and $\mathrm{b}$ is $q_{w}=1 \mathrm{PV} /$ year. Using a lateral

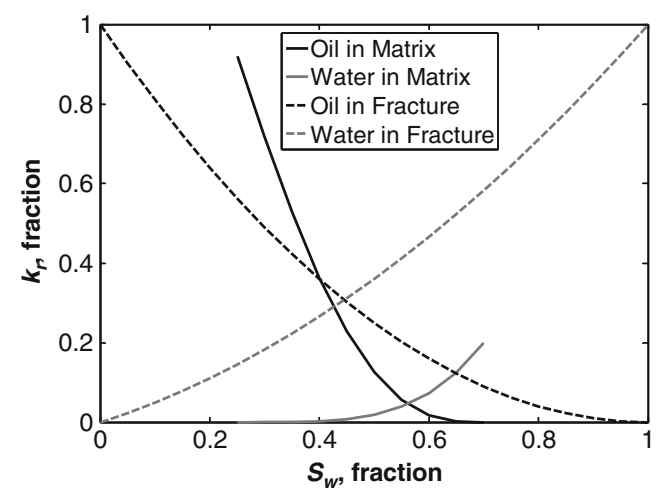

Fig. 5 Relative-permeability curves of matrix and fracture versus water saturation matrix-column size of $\ell=2 \mathrm{~m}$ and a fracture aperture of $100 \mu \mathrm{m}$, the relative error in oil recovery between the fine-grid model and the upscaled-VFR model is $9.2 \%$ for a delay time of $\tau_{b}=3 \times 10^{6} \mathrm{~s}$ at the end of the simulation. For a capillary-damping coefficient of $\tau_{h}=1.8 \times 10^{11} \mathrm{~kg} /(\mathrm{m} \mathrm{s})$ (Fig. $6 \mathrm{~b}$ ), the relative error in oil recovery between the fine-grid simulation and the VFR model is $10.9 \%$ at the end of the simulation. Figure $6 \mathrm{a}$ and $\mathrm{b}$ illustrate only qualitative agreement between the fine-grid and upscaled-VFR model, due to the complex non-equilibrium behavior. Indeed, for $\tau_{h}=1.8 \times 10^{11} \mathrm{~kg} /(\mathrm{m} \mathrm{s})$ (Hassanizadeh), the discrepancy between the fine-grid results and the upscaled-VFR results is larger than for $\tau_{b}=3 \times 10^{6} \mathrm{~s}$ (Barenblatt). Therefore, the numerical dispersion of the upscaled-VFR model in which the non-equilibrium effects are included for the same time and space dis-
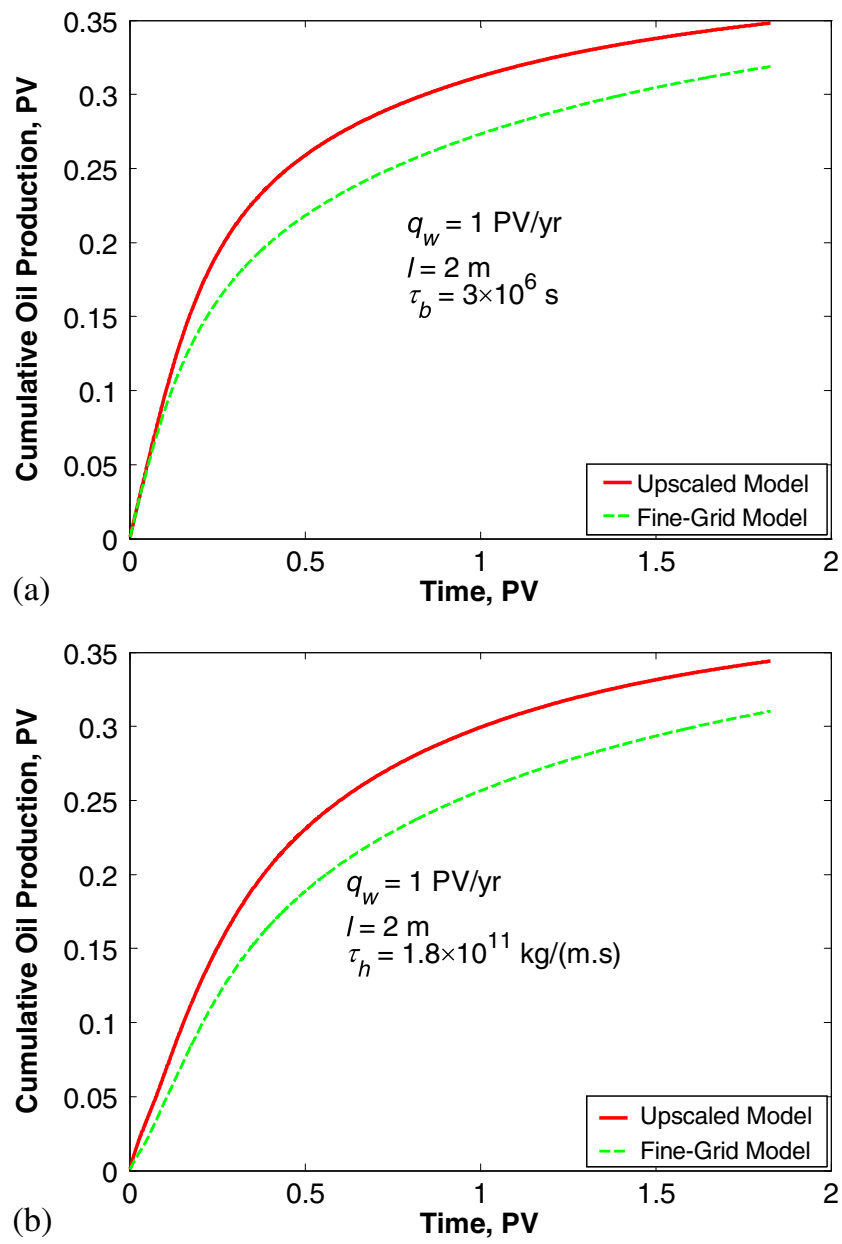

Fig. 6 Comparison between the upscaled model and the finegrid model in terms of the cumulative oil production as a function of PV water injected for $\mathbf{a} q_{w}=1 \mathrm{PV} /$ year, $\ell=2 \mathrm{~m}$, delay time of $\tau_{b}=3 \times 10^{6} \mathrm{~s}$, and $\mathbf{b} q_{w}=1 \mathrm{PV} /$ year, $\ell=2 \mathrm{~m}$, capillary-damping coefficient of $\tau_{h}=1.8 \times 10^{11} \mathrm{~kg} /(\mathrm{m} \mathrm{s})$ 
cretization is larger for higher non-equilibrium effects than for lower non-equilibrium effects. The comparison between the fine-grid results and upscaled-VFR results demonstrates that the upscaled-VFR model can give

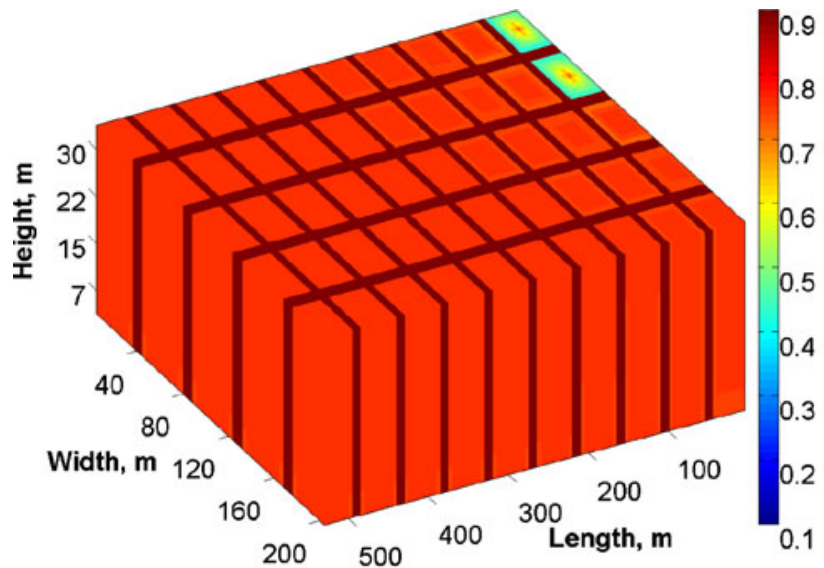

(a)
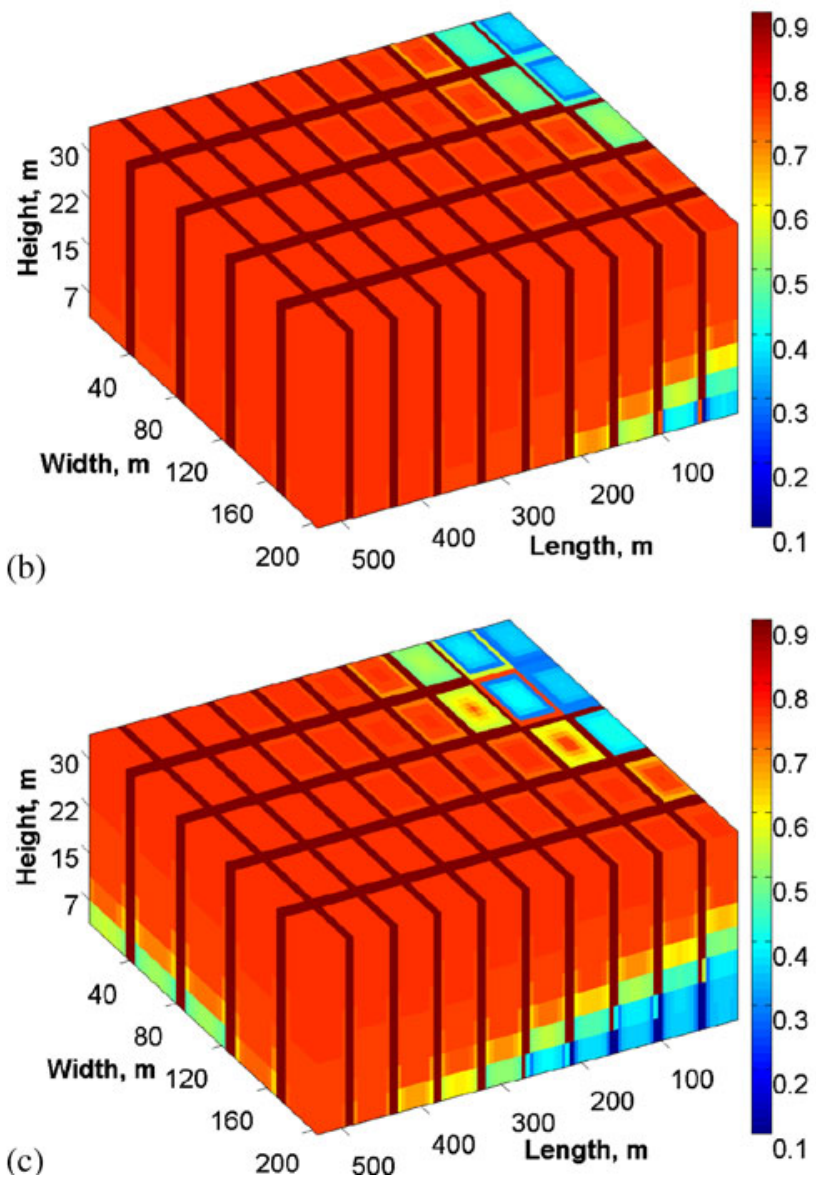

Fig. 7 Oil-saturation history at a $t=50$ days, $\mathbf{b} t=375$ days, $\mathbf{c} t=$ 625 days, $\mathbf{d} t=750$ days, $\mathbf{e} t=1,250$ days, and $\mathbf{f} t=2,000$ days. The reservoir has a length of $500 \mathrm{~m}$ in the $x$-direction, $200 \mathrm{~m}$ in the $y$ direction and $30 \mathrm{~m}$ in the $z$-direction. The water injection rate is physically reasonable results, though some differences between the upscaled-VFR model and the fine-grid model can be observed. The upscaled-VFR model contains 36,900 cells, which represents approximately

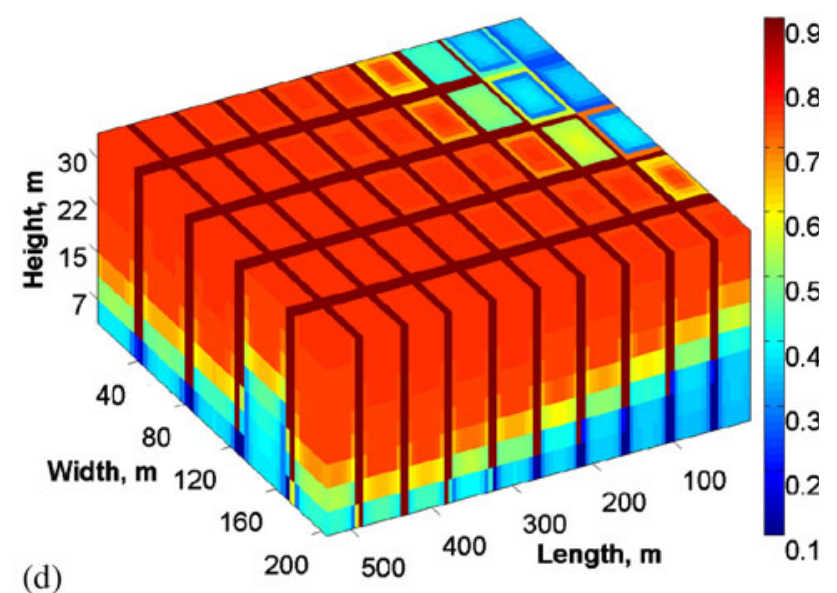

(d)
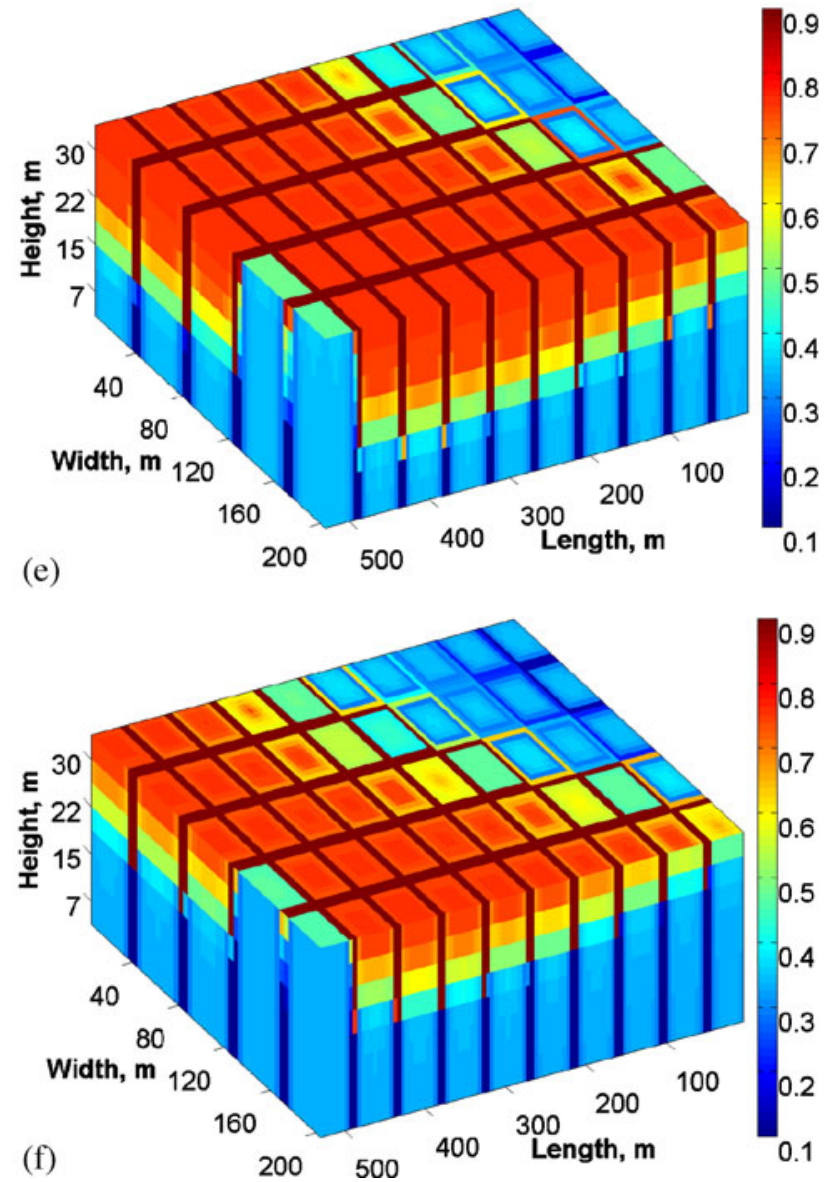

$0.1 \mathrm{PV} /$ year and the lateral matrix-column size is $0.5 \mathrm{~m}$. First, the water occupies the bottom of the reservoir. Then, the water rises in the fractures and in the columns. Due to gravity, the oil at the top of the reservoir is not fully recovered 
a reduction factor of 73 relative to the fine-grid (discrete fracture) model with 2,706,309 grid cells. For the above-mentioned cases, the fine-grid model requires about 14 days, while the upscaled-VFR model needs approximately $10 \mathrm{~h}$. Therefore, the speed up in this case is roughly 34 .

\subsection{Oil-recovery mechanisms}

Figure 7 shows the oil-saturation history after water injection in the two NE corner columns, with production in the top $1 / 3$ of the two SW corner columns. The water saturation first expands via the bottom of the reservoir. In a reservoir that is strongly water-wet, the recovery process in the water-invaded part of the reservoir is a combination of water/oil gravity imbibition and water/oil capillary imbibition.

We observe the following pattern in Fig. 7. First, the water fills the fracture from the bottom because of its higher density. Then, as soon as the water arrives at the fracture/matrix interface, capillary imbibition occurs, the water is invading the matrix column, and oil is produced from the matrix column. Thirdly, because of the distortion of the capillary-gravity equilibrium, a mixture of capillary imbibition and gravity imbibition will occur. In other words, the oil-water capillary-transition zone is moving inside the matrix ahead of the oil/water contact in the fracture system. As a result, oil will leave the matrix columns near the top of the columns and water will enter the matrix columns from the bottom (i.e., water/oil gravity imbibition). Therefore, water/oil gravity imbibition is a co-current imbibition. That is why initially, we see a red rim of oil around many matrix columns in Fig. 7. As more and more water is entering the matrix columns from the bottom via the fracture, the oil saturation becomes less and less and the rim in the fracture grid cells turns increasingly blue. In general, the saturation patterns reflect the strong disparity in capillary pressure between fracture and matrix. Even at relatively high water saturations in the matrix, the oil saturation may still remain high in the surrounding fractures. The columns at the front corner are only perforated at the top third of the reservoir (see Fig. 3), from where the oil (and water) is produced. Due to the production, these two columns are mainly filled with water after 1,250 days. Indeed, we observe in Fig. 7 that co-current (gravity) and counter-current imbibition occur at the same time.

\subsection{Effects of the non-equilibrium}

The purpose of our simulations is to show for which values of the capillary-damping coefficient or delay times, non-equilibrium effects significantly influence the production behavior of fractured reservoirs.

The non-equilibrium behavior changes the capillarypressure and relative-permeability behavior as explained earlier. In Barenblatt's theory, the time to reach equilibrium (delay time) is denoted by $\tau_{b}$, and in Hassanizadeh's theory, the characteristic parameter that indicates the importance of the non-equilibrium effects is the capillary-damping coefficient $\tau_{h}$. Figure 8a shows the effect of the delay time on the cumulative oil production for a water injection rate of $q_{w}=$ $0.1 \mathrm{PV} /$ year and a lateral matrix-column size of $\ell=$ $0.5 \mathrm{~m}$. Here, we describe the oil-recovery ratio between the non-zero delay-time cases and the zero delay-time case. We observe in Fig. 8a that the delay time does not influence oil recovery substantially. The reason is that for low injection rates and thus low Peclet numbers (Fig. $8 \mathrm{a}, P e \approx 0.06$ ), the residence time of water in the fracture (i.e., 913 days) is much longer than any reasonable delay time $\tau_{b}$. The residence time is
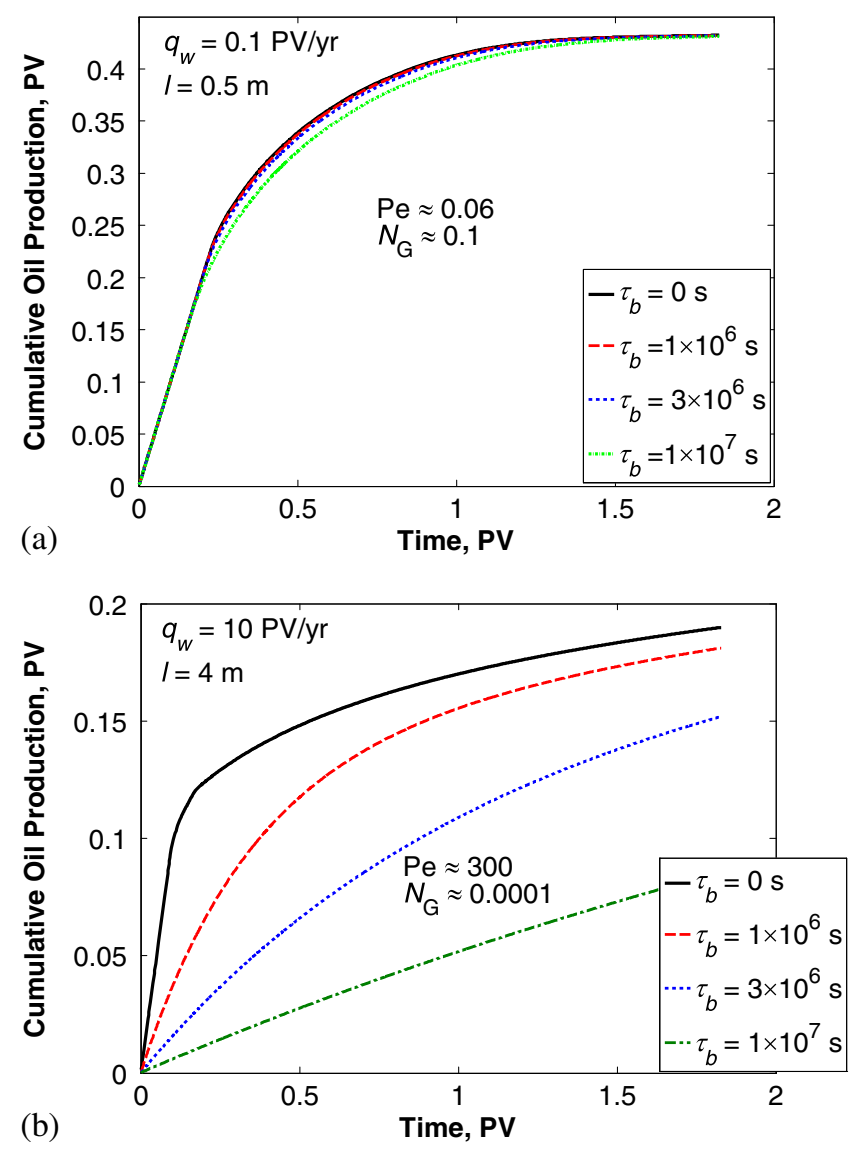

Fig. 8 Effect of the delay time on the cumulative oil production for a water injection rate $q_{w}=0.1 \mathrm{PV} /$ year and lateral matrixcolumn size of $\ell=0.5 \mathrm{~m}$, and $\mathbf{b}$ water injection rate of $q_{w}=10$ $\mathrm{PV} /$ year and lateral matrix-column size of $\ell=4 \mathrm{~m}$ 
the time at which the cumulative-oil-production curve starts to deviate considerably from the initial straight line, indicating significant water breakthrough. In this case, the effect of the delay time is almost zero. On the other hand, we observe from Fig. 8b that at high Peclet numbers, the delay time affects oil production significantly. Moreover, Fig. $8 \mathrm{~b}$ reveals that significant water breakthrough immediately occurs for the nonzero delay-time cases. As a result, the recovery ratio starts decreasing from the beginning until it reaches its minimum value of $0.37,0.16$, and 0.06 , respectively, for $\tau_{b}=10^{6}, 3 \times 10^{6}$, and $10^{7} \mathrm{~s}$ at $t=0.1 \mathrm{PV}$, the time at which water breakthrough occurs for $\tau_{b}=0$. Subsequently, the recovery ratio increases and reaches a value of $0.95,0.80$, and 0.45 , respectively, for $\tau_{b}=$ $10^{6}, 3 \times 10^{6}$, and $10^{7} \mathrm{~s}$ at $t=1.83 \mathrm{PV}$. For high Peclet numbers $(P e \approx 300)$, the residence time of water in the fractures is short, i.e., of the order of $0.1 \mathrm{PV}$ or 3.65 days. In each of the non-zero delay-time cases shown in Fig. 8b, the delay time is much longer than the residence time and thus it influences the cumulative oil production considerably, as expected.

Figure $9 \mathrm{a}$ and $\mathrm{b}$ describe the non-equilibrium effects of Hassanizadeh's theory (capillary-damping coefficient) on the cumulative oil production for a water injection rate of $q_{w}=0.1 \mathrm{PV} /$ year and a lateral matrix-column size of $\ell=0.5 \mathrm{~m}$, and for $q_{w}=$ $10 \mathrm{PV} /$ year and $\ell=4 \mathrm{~m}$, respectively. In the same way as for the delay-time effects (Fig. 8a), Fig. 9a shows that at low Peclet numbers $(P e \approx 0.07)$, the dynamic-capillary pressure for the considered range of the capillary-damping coefficient $\left(\tau_{h}\right)$ between 0 and $10^{11} \mathrm{~kg} /(\mathrm{m} \mathrm{s})$ does not considerably influence the oil recovery. However, Fig. 9b shows that the dynamiccapillary pressure does have noticeable effects on oil recovery and illustrates approximately the same behavior of the non-equilibrium effect for Hassanizadeh's theory as for Barenblatt's theory (Fig. 8b). Values of $\tau_{h}$ and $\tau_{b}$ are comparable in Figs. $8 \mathrm{~b}$ and $9 \mathrm{~b}$ in the linear approximation (see Eq. 6). As revealed in Fig. 9b, the recovery ratio reaches a value of $0.82,0.50$, and 0.21 respectively for $\tau_{h}=6 \times 10^{10}, 1.8 \times 10^{11}$, and $6 \times 10^{11} \mathrm{~kg} /(\mathrm{m} \mathrm{s})$ at $t=1.83 \mathrm{PV}$. Again, the reason for having significant effects of the dynamic-capillary pressure at high Peclet numbers is that the oil-recovery mechanism in this regime is limited by the rate of imbibition from the matrix system.

For intermediate Peclet numbers, we distinguish two cases; one with small $(\ell=0.5 \mathrm{~m})$ and one with large lateral matrix-column size $(\ell=2 \mathrm{~m})$. Figure $10 \mathrm{a}$ and $\mathrm{b}$ show the effect of the delay time on the cumulative oil production for a water injection rate of $q_{w}=1 \mathrm{PV} /$ year and a lateral matrix-column size of $\ell=0.5 \mathrm{~m}$ and
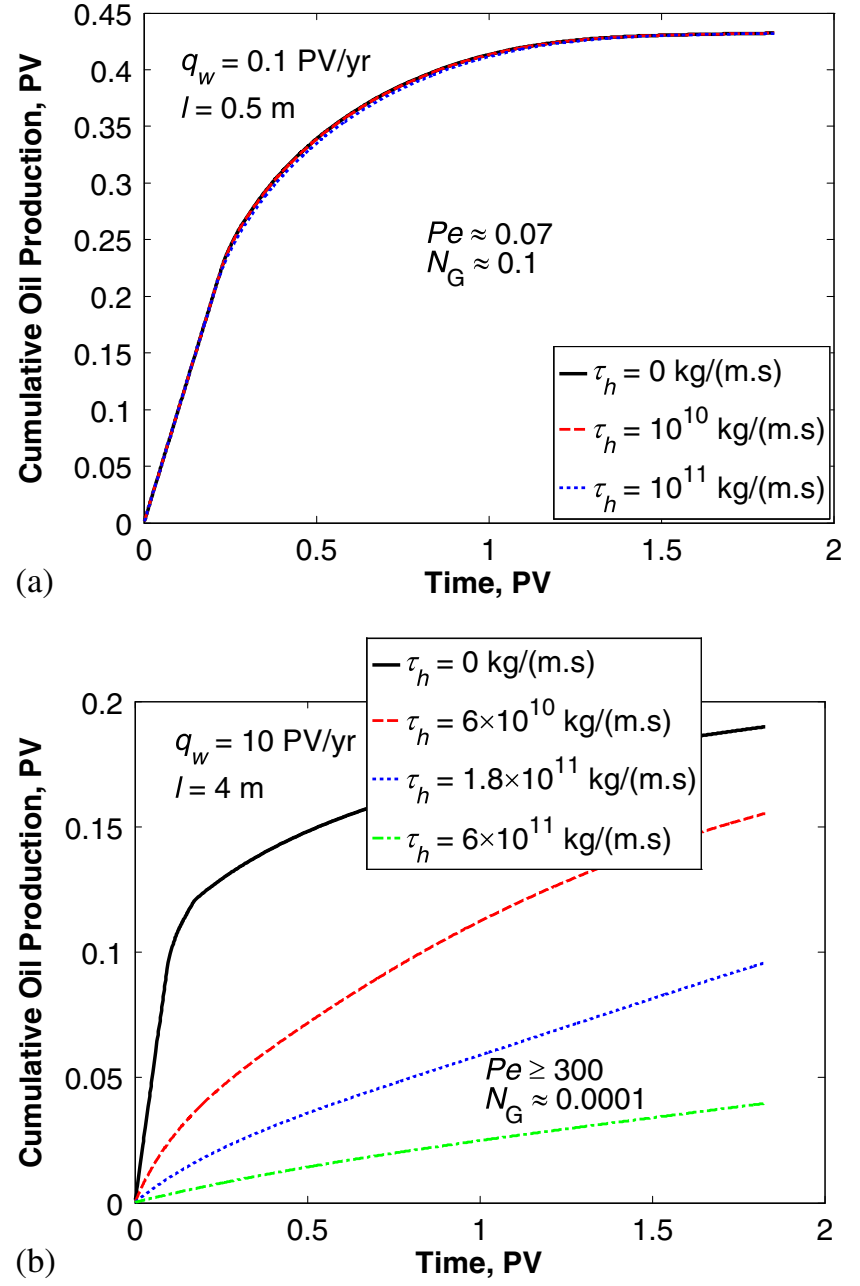

Fig. 9 Effect of the capillary-damping coefficient on the cumulative oil production for a water injection rate $q_{w}=0.1 \mathrm{PV} /$ year and lateral matrix-column size of $\ell=0.5 \mathrm{~m}$, and $\mathbf{b}$ water injection rate of $q_{w}=10 \mathrm{PV} /$ year and lateral matrix-column size of $\ell=4 \mathrm{~m}$

$\ell=2 \mathrm{~m}$, respectively. The size of the matrix columns has an influence on the fracture spacing and hence the global fracture permeability (see Eq. 16). Therefore, this also has an effect on the gravity number $(0.01$ vs. 0.002). Moreover, the size of lateral matrix column influences the Peclet number as well ( 0.3 vs. 1 , see Eq. 36). We observe from Fig. 10a and $\mathrm{b}$ that the residence time of water is about 0.25 PV (90 days) and 0.18 PV (66 days), respectively. This is also reflected by the fact that the Peclet number for the $\ell=0.5 \mathrm{~m}$ case $(P e \approx 0.3)$ is smaller than for the $\ell=2 \mathrm{~m}$ case $(P e \approx 1)$. Figure 10a and $\mathrm{b}$ reveal that the difference between the results for $\tau_{b}=10^{6}$ and $3 \times 10^{6} \mathrm{~s}$ is almost negligible, whereas for the longest delay time $\left(10^{7} \mathrm{~s}\right)$, there is an appreciable effect. For intermediate Peclet numbers, the residence time of the fluids and the delay time are of the same order of magnitude, meaning that for sufficiently long 


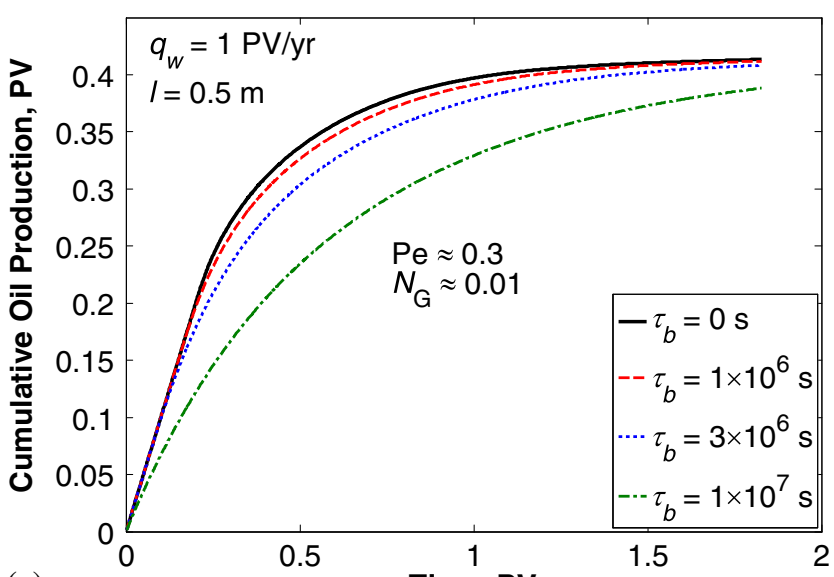

(a)

Time, PV

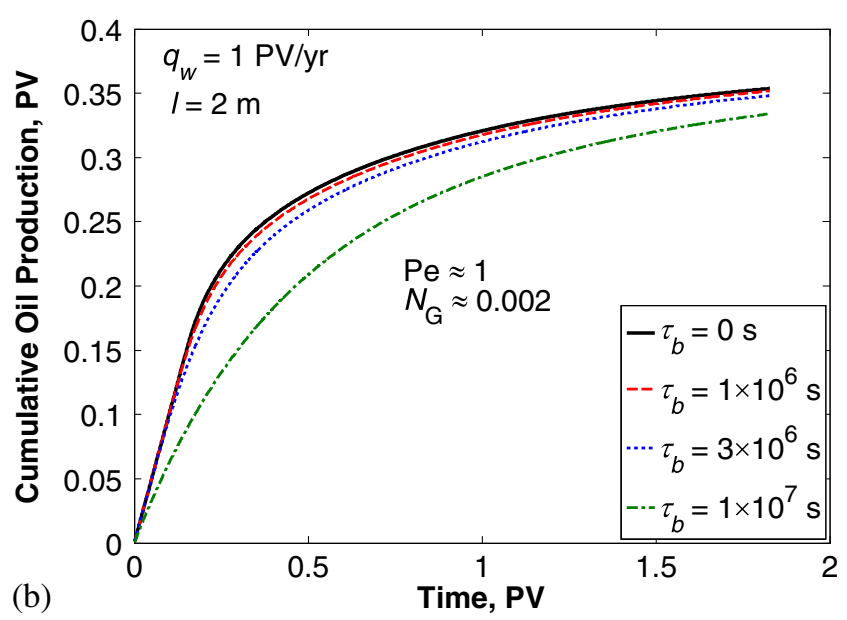

Fig. 10 Effect of the delay time on the cumulative oil production at a water injection rate of $q_{w}=1 \mathrm{PV} /$ year, i.e., at intermediate Peclet numbers, for a lateral matrix-column size of $\ell=0.5 \mathrm{~m}$ and b lateral matrix-column size of $\ell=2 \mathrm{~m}$

delay times $\left(\tau_{b}=10^{7} \mathrm{~s}\right.$ or 116 days), the behavior is completely different from the behavior for short delay times. As shown in Fig. 10a, the results for high gravity numbers show a somewhat larger dependence on the delay time than for small gravity numbers (Fig. 10b).

The general pattern observed in Fig. 10a and b reoccurs in Fig. 11a and $\mathrm{b}$ for the non-equilibrium effects of Hassanizadeh's theory.

To emphasize the effect of the delay time on the displacement mechanism, we show the oil-saturation profiles in Fig. 12a and b for a water injection rate of $q_{w}$ $=1 \mathrm{PV} /$ year without delay time and with a delay time of $\tau_{b}=10^{7} \mathrm{~s}$ at $t=50$ days, respectively. Figure 12a, for zero delay times, clearly illustrates that the waterfront is still far from the production well and water penetrates more into the matrix columns nearby the injection well, while Fig. $12 \mathrm{~b}$ with $\tau_{b}=10^{7} \mathrm{~s}$ describes that water breakthrough has already occurred.

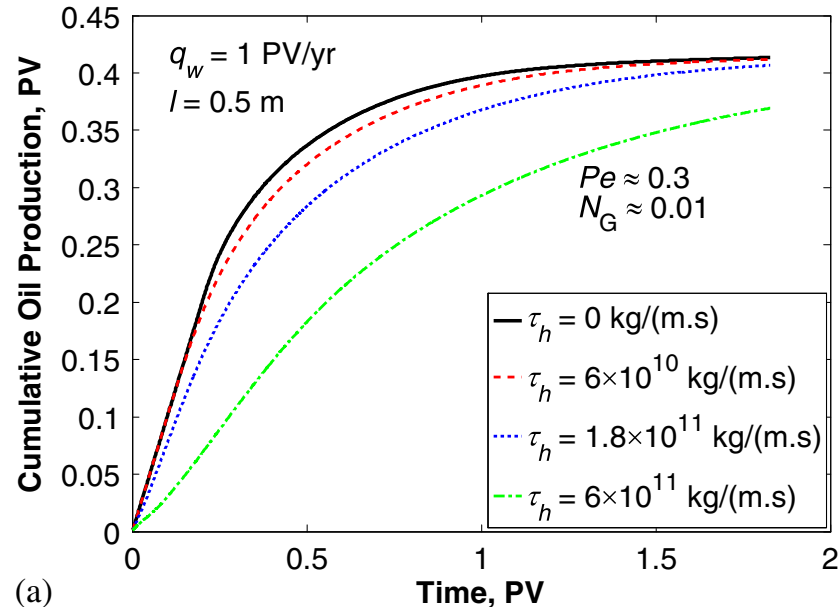

(a)

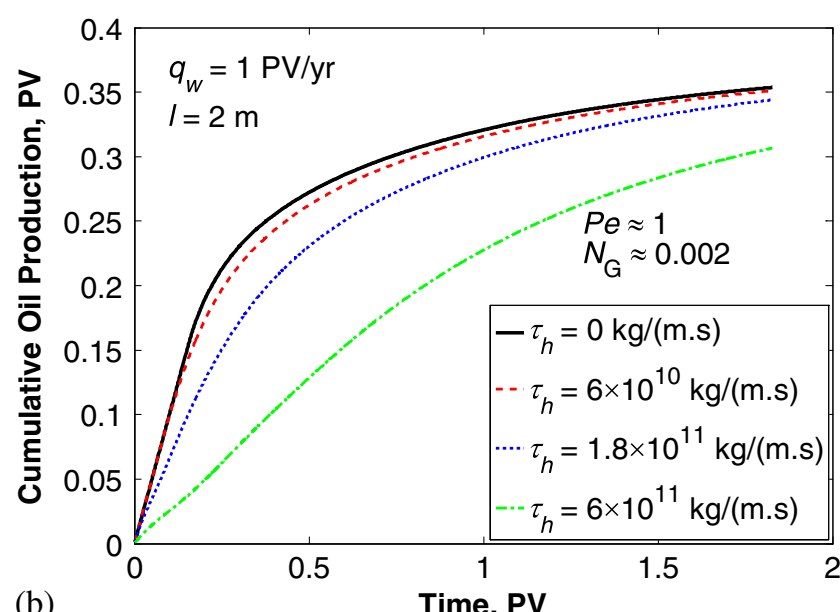

(b)

Time, PV

Fig. 11 Effect of the capillary-damping coefficient on the cumulative oil production at a water injection rate of $q_{w}=1 \mathrm{PV} /$ year, i.e., at intermediate Peclet numbers, for a lateral matrix-column size of $\ell=0.5 \mathrm{~m}$ and $\mathbf{b}$ lateral matrix-column size of $\ell=2 \mathrm{~m}$

\subsection{Comparison between Barenblatt's and Hassanizadeh's approach}

Figure 13a and $\mathrm{b}$ show a comparison of the cumulative oil production between the non-equilibrium approach by Barenblatt and the non-equilibrium approach by Hassanizadeh for a water injection rate of $q_{w}=$ $1 \mathrm{PV} /$ year and a lateral-matrix-column size of $\ell=0.5 \mathrm{~m}$, and for a water injection rate of $q_{w}=10 \mathrm{PV} /$ year and a lateral-matrix-column size of $\ell=4 \mathrm{~m}$, respectively. Figure $13 \mathrm{a}$ and $\mathrm{b}$ present three pairs of cases. Note that for each pair of this comparison, we use Eq. 6 to relate the delay time $\left(\tau_{b}\right)$ of Barenblatt's approach to the capillary-damping coefficient $\left(\tau_{h}\right)$ of Hassanizadeh's approach. Using the matrix-capillary-pressure function shown in Fig. 5, the average capillary derivative $(\Lambda)$ is $60 \mathrm{kPa}$. Figure 13a and b clearly reveal that for each pair of the comparison, the cumulative oil production for 


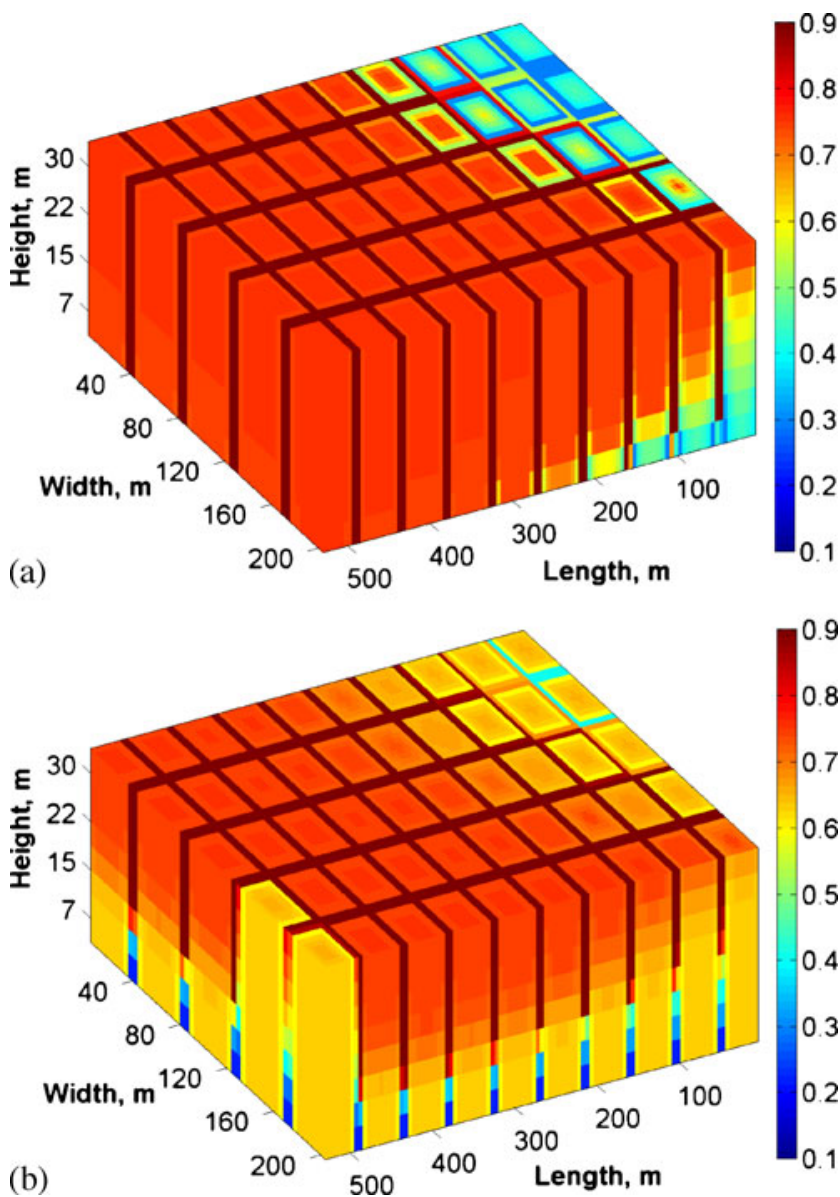

Fig. 12 Oil saturation profiles for a water injection rate of $q_{w}=$ $1 \mathrm{PV} /$ year and a lateral matrix-column size of $\ell=0.5 \mathrm{~m}$ at $t=$ 50 days for Barenblatt's delay time description a $\tau_{b}=0 \mathrm{~s}$ and b $\tau_{b}=10^{7} \mathrm{~s}$

the non-equilibrium effects of Hassanizadeh is larger than that for the non-equilibrium effects of Barenblatt. Moreover, the discrepancy between Barenblatt's and Hassanizadeh's approach for each pair shown in Fig. 13a is smaller than that shown in Fig. 13b. The main reason is that at a smaller lateral matrix-column size of $\ell=0.5 \mathrm{~m}$ and a lower water injection rate $q_{w}=1 \mathrm{PV} /$ year (i.e., Fig. 13a), the corresponding Peclet number is smaller than that for $\ell=4 \mathrm{~m}$ and $q_{w}=10 \mathrm{PV} /$ year (Fig. 13b). As a result, for $\ell=0.5 \mathrm{~m}$ and $q_{w}=1 \mathrm{PV} /$ year, the rate of imbibition is faster and the residence time of fluids in the fracture system is longer. Therefore, the non-equilibrium effects are less pronounced for $\ell=$ $0.5 \mathrm{~m}$ and $q_{w}=1 \mathrm{PV} /$ year as illustrated in Fig. 13a. As the Peclet number increases, the difference between Barenblatt's and Hassanizadeh's approach would also become larger.

Another reason for having discrepancies between Barenblatt's and Hassanizadeh's approach is that the
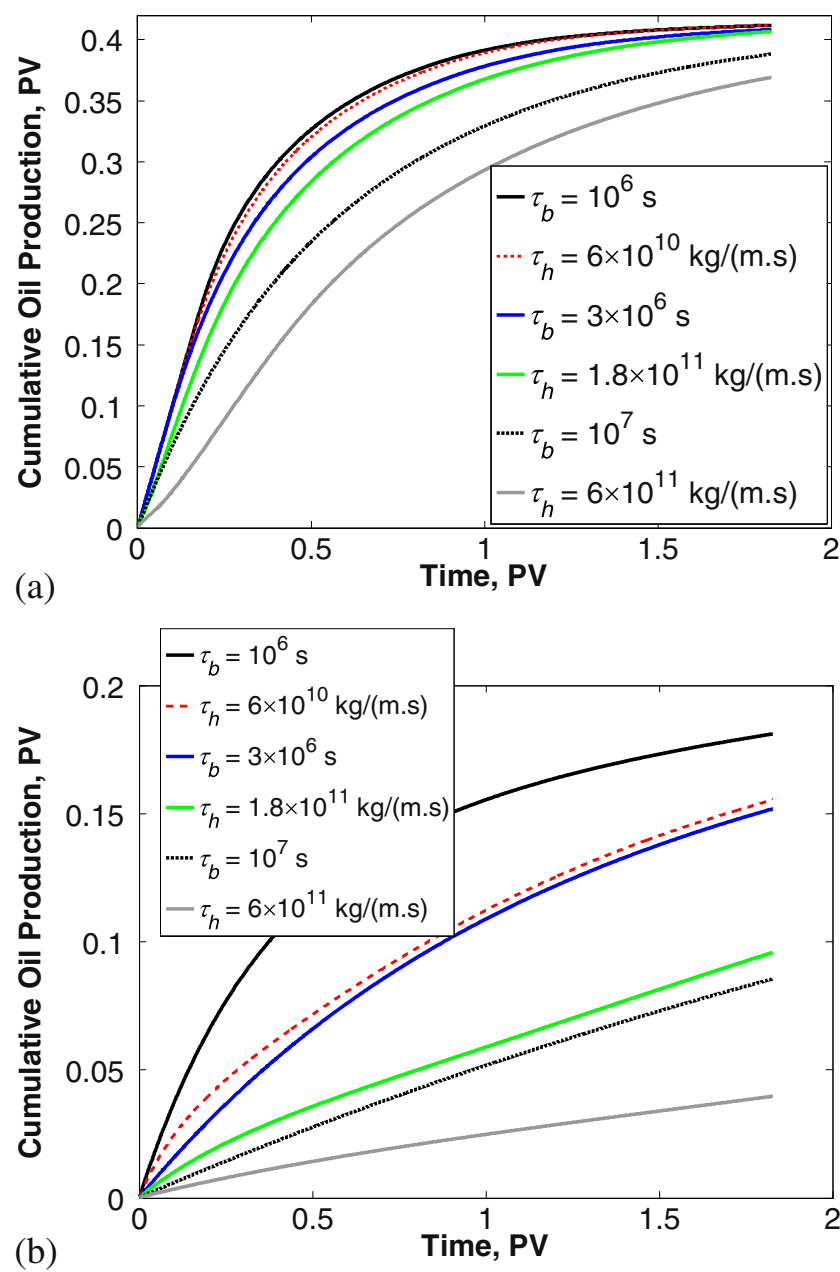

(b)

Fig. 13 Comparison of the cumulative oil production between Barenblatt and Hassanizadeh's theory. a For a water injection rate of $q_{w}=1 \mathrm{PV} /$ year and a lateral matrix-column size of $\ell=$ $0.5 \mathrm{~m}$. b For a water injection rate of $q_{w}=10 \mathrm{PV} /$ year and a lateral matrix-column size of $\ell=4 \mathrm{~m}$. The correspondence of the curves for $\tau_{h}=6 \times 10^{10} \mathrm{~kg} /(\mathrm{m} \mathrm{s})$ and $\tau_{b}=3 \times 10^{6} \mathrm{~s}$ is coincidental. The same can be said about the curves for $\tau_{h}=6$ $\times 10^{11} \mathrm{~kg} /(\mathrm{m} \mathrm{s})$ and $\tau_{b}=3 \times 10^{7} \mathrm{~s}$

non-equilibrium approach of Hassanizadeh does not deal with relative permeabilities, whereas the nonequilibrium theory of Barenblatt modifies relative permeabilities.

In general, the results shown in Fig. 13a and b indicate the fact that the non-equilibrium theory of Hassanizadeh cannot be related to Barenblatt's approach if constant values of $\tau_{b}$ and $\tau_{h}$ are used. Even if varying values of $\tau_{b}$ and $\tau_{h}$ were used, it is almost impossible to obtain a relation between the delay time $\left(\tau_{b}\right)$ and the capillary-damping coefficient $\left(\tau_{b}\right)$ to be able to get quantitatively the same results; because (1) capillary pressures are always non-linear functions and (2) the non-equilibrium effects of relative permeabilities of Barenblatt's approach do not exist in Hassanizadeh's 
theory. However, both approaches illustrate qualitatively the importance of the non-equilibrium effects in terms of oil recovery from fractured reservoirs.

\subsection{Laboratory experiments}

The non-equilibrium effects have consequences for laboratory experiments.

It can be argued that delay times are caused by nonequilibrium effects on the micro-scale based on the following observations: measurements of contact angles show that considerable equilibrium times are required before an equilibrium contact angle is obtained [41]. In this case, the surface is in direct contact with the relevant fluids. Moreover, in contact angle measurements the surface is flat. Capillary measurements in oilwater-solid systems show a positive capillary pressure for a drainage curve, but often a negative pressure for imbibition [34, 35]. This behavior, like in the contactangle measurements, can be attributed to the surface replacement of the non-wetting phase by the wetting phase.

Typical mechanisms are dissolution of the nonwetting phase in the wetting phase and subsequent diffusion. Such processes are known to be very time consuming. In summary, the characteristic times are very long, due to microscopic processes. The theories of Barenblatt and Hassanizadeh can be considered as a continuum approach to describe these microscopic effects.

In the laboratory, a typical cylindrical core has a length of $20 \mathrm{~cm}$ and a diameter of $10 \mathrm{~cm}$. In addition, a typical injection rate is $5 \mathrm{ml} / \mathrm{min}$. To be able to calculate the Peclet number, we need to estimate the capillarydiffusion coefficient. We assume that the absolute permeability of the core is $1 \mathrm{mD}$. Moreover, we use the matrix-relative-permeability functions that are plotted in Fig. 5, and the average capillary derivative and the average mobility factor $\left[\lambda_{o} \lambda_{w} /\left(\lambda_{o}+\lambda_{w}\right)\right]$ for calculating $D_{\text {cap }}$. Note that the characteristic lengths (matrix size) for the capillary diffusion and convection transport (core length) in a laboratory core are the same. Therefore, the definition for the Peclet number in Eq. 36 reduces to $P e_{l a b}=U_{i n j} L / D_{\text {cap }}$. For the laboratory specifications mentioned above, the calculated Peclet number would be $P e_{l a b}=1,768$. Therefore, most of the laboratory experiments are in the high-Peclet-number regime. As a result, the non-equilibrium behavior has a considerable effect on the rate of oil production. Consequently, in the laboratory, samples with long delay times and/or large capillary-damping coefficients could be erroneously considered oil-wet. This would have consequences for any remediative action $[38,39]$ to enhance the imbibition rates.

\subsection{Summary}

The salient features of the upscaled-VFR model including the non-equilibrium effects are summarized in Fig. 14, where the cumulative oil production at $1 \mathrm{PV}$ is plotted versus the Peclet number $(\mathrm{Pe})$ and the gravity number $\left(N_{G}\right)$ for various delay times. At small gravity
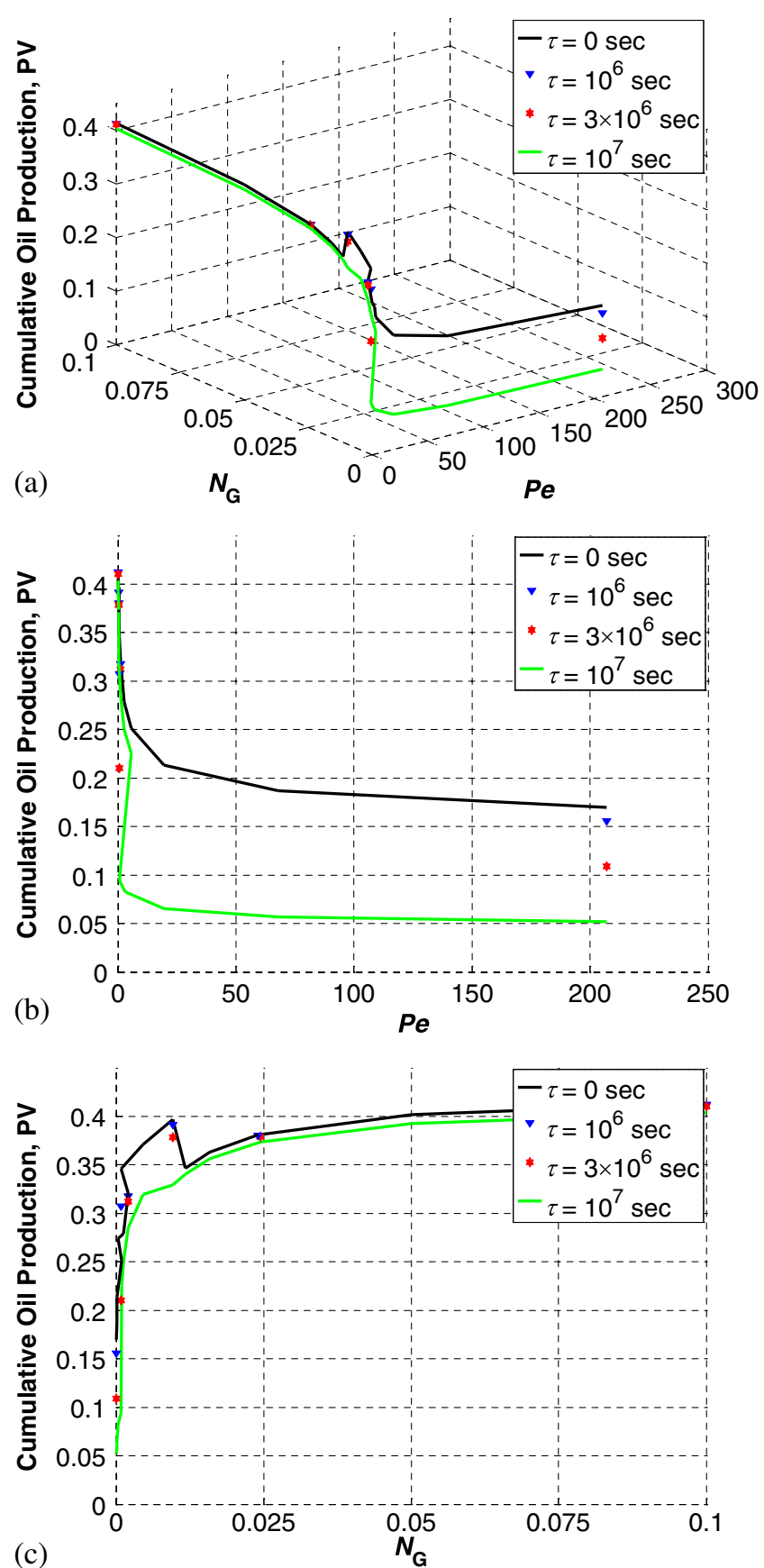

Fig. 14 Cumulative oil production at one PV injected water a versus the gravity number $\left(N_{G}\right)$ and the Peclet number $(P e)$, b projected on the cumulative oil production-Peclet plane, $\mathbf{c}$ projected on the cumulative oil production-gravity number plane 
numbers and large Peclet numbers, the recovery is low. The recovery at small gravity numbers is rather insensitive to the Peclet number. As the gravity number increases and the Peclet number decreases, the recovery becomes much higher. It can be expected that here counter-current imbibition is replaced by the much more effective co-current gravity imbibition. Also, the rate of imbibition from the matrix column becomes larger compared to the rate of fluid transport in the fracture system. In the absence of delay times, the maximum recovery becomes higher than the recoveries where delay times are relevant. At a critical value of the gravity number-Peclet number, there is a sudden decrease in oil recovery when delay times are ignored. However, there is a smooth transition between the two regimes, from small to large Peclet numbers. At high Peclet numbers, the rate of oil production becomes limited by imbibition and hence by the delay time. One may speculate that the transition from where the result is independent of the imbibition rate, due to long residence times of fluids in the fracture, to the situation at large Peclet numbers where it does become sensitive to the imbibition rate is unstable. This feature requires further study.

\section{Conclusions}

- We derive a physically based upscaled model in which the non-equilibrium effects are included for the VFR using homogenization.

- We develop a computationally efficient numerical scheme to solve the upscaled-VFR model.

- At low Peclet numbers, the rate of capillary imbibition dominates over the convective transport in the fracture. Therefore, the characteristic time of fluidflow exchange at the boundary between fracture and matrix is short with respect to the residence time of water in the fracture. Hence, the water cut is small.

- At high Peclet numbers, the convective transport is dominant over capillary imbibition and hence the oil-recovery mechanism is controlled by the rate of imbibition. Furthermore, the residence time of water is much shorter than the characteristic time of imbibition. As a result, less fluid-flow exchange occurs for the same amount of injected water. Thus, if we were in the high-Peclet-number regime, the recovery would be low accordingly. Hence, the water cut is large.

- The rate of capillary imbibition can also be reduced due to non-equilibrium effects, which are described by delay times $\tau_{b}$ or capillary-damping coefficients $\tau_{h}$. If the residence time of water in the fractured reservoir is much longer than the delay time $\tau_{b}$, the delay time (non-equilibrium effects) does not influence oil recovery qualitatively. Conversely, for large Peclet numbers, the residence time of water in the fractures is short and hence high values of $\tau_{b}$ and $\tau_{h}$ significantly slow down the oil recovery due to the non-equilibrium effects.

- In general, due to the non-linear nature of the capillary-pressure function, it is not possible to find a relation between the delay time $\tau_{b}$ (Barenblatt) and the capillary-damping coefficient $\tau_{h}$ (Hassanizadeh) and consequently to make a direct comparison between the two descriptions. Even if the results between the description of Barenblatt and Hassanizadeh cannot be directly compared, the qualitative behavior is the same and both approaches show the possible importance of taking into account non-equilibrium effects in the capillary-pressure and relative-permeability behavior.

- It is asserted that in partially water-wet systems, the values of the delay time $\tau_{b}$ can be long (of the order of 100 days) because first an oil film must be removed from the corrugated surface of the grains by a combined dissolution/diffusion process. Correspondingly, also the capillary-damping coefficient $\tau_{h}$ can be high.

- Experiments in the laboratory are expected to be more sensitive to non-equilibrium capillarypressure effects as Peclet numbers are usually high. The delay time $\tau_{b}$ can easily exceed the experimentation time.

In view of the transparent physical basis of homogenization, we assert that improved fracture modeling can be achieved using the upscaled-VFR model. Based on the comparison between the fine-grid model and the upscaled-VFR model, the upscaled-VFR model is capable to predict oil recovery adequately.

Acknowledgements We thank Statoil-Hydro for supporting our research on oil recovery from fractured reservoirs. We also thank Sharif University of Technology for their steady collaboration.

Open Access This article is distributed under the terms of the Creative Commons Attribution Noncommercial License which permits any noncommercial use, distribution, and reproduction in any medium, provided the original author(s) and source are credited. 


\section{Nomenclature}

A

$B$

$D_{\text {cap }}$

F

$F_{1,2}$

$\mathrm{g}$

H

I

$J$

$k$

$k_{r}$

l

$L$

M

n

N

$N_{G}$

$o$

$P$

$P_{c}$

$P e$

PV

q

$Q$

$q_{\text {ext }}$

$q_{w}$

r

$\mathbf{r}^{\prime}$

$R$

$S$

$S_{\text {or }}$

$S_{w, i r}$

SU

$t$

$\mathrm{u}$

$\mathbf{u}$

$w$

W

$x$

$y$

$z$

$\mathrm{Z}$

\section{Greek}

\section{$\alpha$}

$\varepsilon$
Horizontal cross-section

3D domain

Capillary-diffusion

coefficient

Nonlinear fracture function

Fracture set

Gravity acceleration

Height of the reservoir

Unit tensor

Leverett $J$-function

Permeability

Relative permeability

Local scale (lateral matrix-

column size)

Global scale/length of the reservoir

Nonlinear matrix function

Unit normal vector

Number of fracture grid cells

Gravity number

Oil

Pressure

Capillary pressure

Peclet number

Pore volume

Any vector

3D domain

Injection/production rate

Water injection rate

Coordinate vector

Vector of the cell center on

a horizontal cross-section

Real numbers/radius

Saturation

Residual oil saturation

Irreducible water saturation

Small unit

Time

Velocity

Velocity vector

Water

Width of the reservoir

$\mathrm{x}$-coordinate

$y$-coordinate

Vertical upward direction

1D domain

Phase (oil/water)

Scaling ratio
$\Lambda$

$\mu$

$\xi$

$\rho$

$\sigma$

$\tau_{b}$

$\tau_{h}$

$\varphi$

$\Phi$

$\Omega$

$\omega$

\section{Math Signs and Operator}

II

$\sim$

$\approx$

$\otimes$

$\cup$

$\epsilon$

$\rightarrow$

$\mathrm{d}$

$\partial_{\pi}$

$\partial$

$\nabla$

$\Delta$

$\nabla$.

\section{Subscripts}

$b$

D

$f$

inj

$l a b$

$m$

$o$

$r$

$\mathrm{R}$

S

$w$

z

$\alpha$

$\zeta$

Superscripts

b
Effective water saturation

Mobility

Constant coefficient

Viscosity

Potential/saturation

indicator

Density

Surface tension

Delay time

Capillary-damping

coefficient

Porosity

Potential

Horizontal cross-section domain

Auxiliary function

Absolute value of volume

Same order of magnitude

Almost equal to

Dyadic tensor product

The union of

An element of

Vector sign

Differential

Partial differential with respect to $\pi$

Boundary of

Del (gradient operator)

Delta (difference operator)

Divergence operator

Global (big) index

Dimensionless

Fracture

Injection

Laboratory

Matrix

Oil phase

Relative

Reference

Local (small) index

Water phase

z-coordinate

(vertical direction)

Oil/water index

Fracture/matrix index

Barenblatt's approach index 


$\begin{array}{ll}d y n & \text { Dynamic } \\ h & \text { Hassanizadeh's approach } \\ & \text { index } \\ k & \mathrm{k}^{\text {th }} \text { iteration } \\ n & \mathrm{n}^{\text {th }} \text { time level } \\ \text { stat } & \text { Static } \\ * & \text { Local (intrinsic) fracture } \\ & \text { index } \\ 0 & \text { Initial guess }\end{array}$

\section{References}

1. Aguilera, R.: Geological aspects of naturally fractured reservoirs. The Leading Edge 17(12), 1667-1670 (1998)

2. Al-Harbi, M., Cheng, H., He, Z., Datta-Gupta, A.: Streamline-based production data integration in naturally fractured reservoirs. SPE J. 10(4), 426-439 (2005)

3. Al-Huthali, A., Datta-Gupta, A.: Streamline simulation of counter-current imbibition in naturally fractured reservoirs. J. Pet. Sci. Eng. 43(3-4), 271-300 (2004)

4. Anderson, W.G.: Wettability literature survey-part 6: the effects of wettability on waterflooding. J. Pet. Technol. 39(12), 1605-1622 (1987)

5. Arbogast, T.: Computational aspects of dual-porosity models. In: Hornung, U. (ed.) Homogenization and Porous Media, Interdisciplinary Applied Mathematics, 6th edn., pp. 203-223. Springer, New York (1997)

6. Barenblatt, G.I.: Filtration of two nonmixing fluids in a homogeneous porous medium. Fluid Dyn. 6(5), 857-864 (1974). doi:10.1007/BF01013869

7. Barenblatt, G.I., Gilman, A.A.: Nonequilibrium counterflow capillary impregnation. J. Eng. Phys. 52(3), 335-339 (1987)

8. Barenblatt, G.I., Zheltov, Iu.P., Kochina, I.N.: Basic concepts in the theory of seepage of homogeneous liquids in fissured rocks. J. Appl. Math. Mech. 24(5), 1286-1303 (1960). doi:10.1016/0021-8928(60)90107-6

9. Barenblatt, G.I., Garcia-Azorero, J., De Pablo, A., Vazquez, J.L.: Mathematical model of the non-equilibrium water-oil displacement in porous strata. Appl. Anal. 65, 19-45 (1997)

10. Barenblatt, G.I., Patzek, T.W., Silin, D.B.: The mathematical model of nonequilibrium effects in water-oil displacement. SPE J. 8(4), 409-416 (2003)

11. Behbahani, H., Blunt, M.J.: Analysis of imbibition in mixedwet rocks using pore-scale modeling. SPE J. 10(4), 466-473 (2005)

12. Dahan, O., Nativ, R., Adar, E., Berkowitz, B.: A measurement system to determine water flux and solute transport through fractures in the unsaturated zone. Ground Water 36(3), 444-449 (1998)

13. Douglas, J., Jr., Hensley, J.L., Arbogast, T.: A dual-porosity model for waterflooding in naturally fractured reservoirs. Comput. Methods Appl. Mech. Eng. 87(2-3), 157-174 (1991)

14. Elzeftawy, A., Mansell, R.S.: Hydraulic conductivity calculations for unsaturated steady-state and transient-state flow in sands. Soil Sci. Soc. Am. Proc. 39, 599-603 (1975)

15. Finkbeiner, T., Barton, C.A., Zoback, M.D.: Relationships among in-situ stress, fractures and faults, and fluid flow: Monterey formation, Santa Maria basin, California. A.A.P.G. Bulletin 81(12), 1975-1999 (1997)

16. Firoozabadi, A.: Recovery mechanisms in fractured reservoirs and field performance. J. Can. Pet. Technol. 39(11), 13$17(2000)$
17. Ghorayeb, K., Firoozabadi, A.: Numerical study of natural convection and diffusion in fractured porous media. SPE J. 5(1), 12-20 (2000)

18. Graue, A., Bogn $\varnothing$, T., Baldwin, B.A., Spinler, E.A.: Wettability effects on oil-recovery mechanisms in fractured reservoirs. SPE Reserv. Evalu. Eng. 4(6), 455-465 (2001)

19. Hassanizadeh, S.M., Gray, W.G.: Mechanics and thermodynamics of multiphase flow in porous media including interphase boundaries. Adv. Water Resour. 13(4), 169-186 (1990)

20. Hassanizadeh, S.M., Gray, W.G.: Thermodynamic basis of capillary pressure in porous media. Water Resour. Res. 29(10), 3389-3405 (1993)

21. Hassanizadeh, S.M., Celia, M.A., Dahle, H.K.: Dynamic effect in the capillary pressure-saturation relationship and its impact on unsaturated flow. Vadose Zone J. 1, 38-57 (2002)

22. Hatiboglu, C.U., Babadagli, T.: Oil recovery by countercurrent spontaneous imbibition: Effects of matrix shape factor, gravity, IFT, oil viscosity, wettability, and rock type. J. Pet. Sci. Eng. 59(1-2), 106-122 (2007)

23. Hirasaki, G.J.: Wettability: fundamentals and surface forces. SPE Form. Eval. 6(2), 217-226 (1991)

24. Hirasaki, G., Zhang, D.L.: Surface chemistry of oil recovery from fractured, oil-wet, carbonate formations. SPE J. 9(2), 151-162 (2004)

25. Karimaie, H., Torsæter, O.: Effect of injection rate, initial water saturation and gravity on water injection in slightly water-wet fractured porous media. J. Pet. Sci. Eng. 58(1-2), 293-308 (2007)

26. Kazemi, H.: The interpretation of interference tests in naturally fractured reservoirs with uniform fracture distribution. SPE J. 9(4), 451-462 (1969)

27. Kazemi, H., Gilman, J.R., Elasharkawy, A.M.: Analytical and numerical solution of oil recovery from fractured reservoirs with empirical transfer functions. SPE Reserv. Eng. 7(2), 219-227 (1992)

28. Landau, L.D., Lifshitz, E.M.: Fluids Mechanics, 2nd edn. Pergamon, Oxford, England (1987)

29. Leverett, M.C.: Flow of oil-water mixtures through unconsolidated sands. Trans., AIME 132, 149-171 (1939)

30. Morrow, N.R., Mason, G.: Recovery of oil by spontaneous imbibition. Curr. Opin. Colloid Interface Sci. 6(4), 321-337 (2001)

31. Muskat, M., Meres, M.W.: The flow of heterogeneous fluids through porous media. J. Appl. Phys. 7(921), 346-363 (1936)

32. Nelson, R.A.: Geologic Analysis of Naturally Fractured Reservoirs, 2nd edn. Gulf Professional, Woburn, USA (2001)

33. Pavone, D.R.: A Darcy's law extension and a new capillary pressure equation for two-phase flow in porous media. Paper SPE 20474 presented at the SPE Annual Technical Conference and Exhibition, New Orleans, Louisiana, 23-26 September, (1990). doi:10.2118/20474-MS

34. Plug, W.J., Bruining, J.: Capillary pressure for the sand-- $\mathrm{CO}_{2}-$ water system under various pressure conditions. Application to $\mathrm{CO}_{2}$ sequestration. Adv. Water Resour. 30(11), 2339-2353 (2007)

35. Plug, W.J., Mazumder, S., Bruining, J.: Capillary pressure and wettability behavior of $\mathrm{CO}_{2}$ sequestration in coal at elevated pressures. SPE J. 13(4), 455-464 (2008)

36. Pooladi-Darvish, M., Firoozabadi, A.: Cocurrent and countercurrent imbibition in a water-wet matrix block. SPE J. 5(1), 3-11 (2000)

37. Price, H.S., Coats, K.H.: Direct methods in reservoir simulation. SPE J. 14(3), 295-308 (1974) 
38. Puntervold, T., Austad, T.: Injection of seawater and mixtures with produced water into North Sea chalk formation: impact of fluid-rock interactions on wettability and scale formation. J. Pet. Sci. Eng. 63(1-4), 23-33 (2008)

39. Puntervold, T., Strand, S., Austad, T.: Coinjection of seawater and produced water to improve oil recovery from fractured North Sea chalk oil reservoirs. Energy Fuels 23(5), 25272536, (2009)

40. Rangel-German, E.R., Kovscek, A.R.: Experimental and analytical study of multidimensional imbibition in fractured porous media. J. Pet. Sci. Eng. 36(1-2), 45-60 (2002)

41. Saidi, A.M.: Simulation of naturally fractured reservoirs. Paper SPE 12270 presented at the SPE Reservoir Simulation Symposium, San Francisco, California, 15-18 November (1983). doi:10.2118/12270-MS

42. Salimi, H., Bruining, J.: Improved prediction of oil recovery from waterflooded fractured reservoirs. Paper SPE 115361 presented at the SPE Annual Technical Conference and Exhibition, Denver, Colorado, 21-24 September (2008). doi:10.2118/115361-MS

43. Salimi, H., Bruining, J.: Upscaling in partially fractured oil reservoirs using homogenization. Paper SPE 125559 presented at the SPE/EAGE Reservoir Characterization and Simulation Conference, Abu Dhabi, UAE, 19-21 October (2009). doi:10.2118/125559-MS

44. Salimi, H., Bruining, J.: Improved prediction of oil recovery from waterflooded fractured reservoirs using homogenization. SPE Reserv. Evalu. Eng. 13(1), 44-55 (2010a). doi:10.2118/115361-PA

45. Salimi, H., Bruining, H.: Upscaling in vertically fractured oil reservoirs using homogenization. Transp. Porous Media. 84(1), 21-53 (2010b)

46. Salimi, H., Bruining, J.: Upscaling in partially fractured oil reservoirs using homogenization. SPE J. 16(2), 273-293 (2011a). doi:10.2118/125559-PA

47. Salimi, H., Bruining, J.: The influence of heterogeneity, wettability, and viscosity ratio on oil recovery from vertically fractured oil reservoirs. SPE J. 16(2), 411-428 (2011b). doi:10.2118/140152-PA

48. Sarma, P., Aziz, K.: New transfer function for simulation of naturally fractured reservoirs with dual-porosity models. SPE J. 11(3), 328-340 (2006)

49. Seethepalli, A., Adibhatla, B., Mohanty, K.K.: Physicochemical interactions during surfactants flooding of fractured carbonate reservoirs. SPE J. 9(4), 411-418 (2004)

50. Shook, M., Li, D., Lake, L.W.: Scaling immiscible flow through permeable media by inspectional analysis. In SITU 16(4), 311-349 (1992)
51. Siemons, N., Bruining, J., Castelijns, H., Wolf, K.H.: Pressure dependence of the contact angle in a $\mathrm{CO}_{2}-\mathrm{H}_{2} \mathrm{O}-$ coal system. J. Colloid Interface Sci. 297(2), 755-761 (2006)

52. Smiles, D.E., Vachaud, G., Vauclin, M.: A test of the uniqueness of the soil moisture characteristic during transient, nonhysteretic flow of water in a rigid soil. Soil Sci. Soc. Am. Proc. 35, 535-539 (1971)

53. Sonier, F., Souillard, P., Blaskovich, F.T.: Numerical simulation of naturally fractured reservoirs. SPE Reserv. Eng. 3(4), 1114-1122 (1988)

54. Stauffer, F.: Time dependence of the relations between capillary pressure, water content and conductivity during drainage of porous media. IAHR Symposium on Scale Effects in Porous Media, Thessaloniki, Greece, 29 August-1 September (1978)

55. Stringer, J.C., Thomas, L.K., Pierson, R.G.: Efficiency of D4 Gaussian elimination on a vector computer. SPE J. 25(1), 121-124 (1985)

56. Tang, G.-Q., Firoozabadi, A.: Effect of pressure gradient and initial water saturation on water injection in water-wet and mixed-wet fractured porous media. SPE Reserv. Evalu. Eng. 4(6), 516-524 (2001)

57. Teixell, A., Durney, D.W., Arboleya, M.L.: Stress and fluid control on decollement within competent limestone. J. Struct. Geol. 22(3), 349-371 (2000)

58. Topp, G.C., Klute, A., Peters, D.B.: Comparison of water content-pressure head data obtained by equilibrium, steadystate and unsteady-state methods. Soil Sci. Soc. Am. Proc. 31, 312-314 (1967)

59. Vachaud, G., Vauclin, M., Wakil, M.: A study of the uniqueness of the soil moisture characteristic during desorption by vertical drainage. Soil Sci. Soc. Am. Proc. 36, 531-532 (1972)

60. Van Duijn, C.J., Molenaar, J. Neef, M.J.: The effect of capillary forces on immiscible two-phase flow in heterogeneous porous media. Transp. Porous Media 21(1), 71-93 (1995)

61. Warren, J.E., Root, P.J.: The behavior of naturally fractured reservoirs. SPE J. 3(3), 245-255 (1963)

62. Yortsos, Y.C.: A theoretical analysis of vertical flow equilibrium. Transp. Porous Media 18(2), 107-129 (1995)

63. Yu, L., Evje, S., Kleppe, H., Karstad, T., Fjelde, I., Skjaeveland, S.M.: Spontaneous imbibition of seawater into preferentially oil-wet chalk cores-experiments and simulations. J. Pet. Sci. Eng. 66(3-4), 171-179 (2009)

64. Zanini, L., Novakowski, K.S., Lapcevic, P., Bickerton, G.S., Voralek, J., Talbot, C.: Ground water flow in a fractured carbonate aquifer inferred from combined hydrogeological and geochemical measurements. Ground Water 38(3), 350$360(2000)$ 